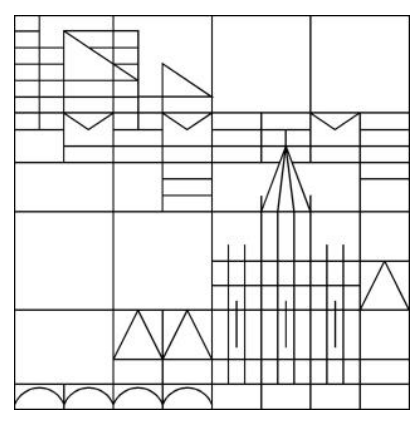

\title{
Stability of Abstract Thermoelastic Systems with Inertial Terms
}

\author{
Hugo D. Fernández Sare \\ Zhuangyi Liu \\ Reinhard Racke
}

Konstanzer Schriften in Mathematik

Nr. 376, Mai 2018

ISSN 1430-3558

Konstanzer Online-Publikations-System (KOPS)

URL: http://nbn-resolving.de/urn:nbn:de:bsz:352-2-1 fwby15urvlbl0

(C) Fachbereich Mathematik und Statistik

Universität Konstanz

Fach D 197, 78457 Konstanz, Germany 



\section{Stability of Abstract Thermoelastic Systems with Inertial Terms}

\section{Hugo D. Fernández Sare, Zhuangyi Liu and Reinhard Racke}

Abstract: We investigate coupled systems of thermoelastic type in a general abstract form both modeling Fourier and Cattaneo type heat conduction. In particular we take into account a possible inertial term. A complete picture of the regions of exponential stability resp. non-exponential stability for the arising parameters (two arising from the type of thermoelastic system, one arising from the inertial term) is given. The regions of loss of exponential stability, while moving from the Fourier to the Cattaneo law, are thus clearly recognized and interestingly large. The polynomial stability in regions of non-exponential stability is also characterized.

\section{Introduction}

For given parameters $\mu>0,(\alpha, \beta) \in[0,1] \times[0,1]$ and $\gamma \in(0,1]$ and $A$ a self-adjoint, positive definite operator on a Hilbert space $H$, we first consider the abstract thermoelastic system given by

$$
\begin{aligned}
\rho u_{t t}+\mu A^{\gamma} u_{t t}+\sigma A u-m A^{\alpha} \theta & =0, \\
c \theta_{t}+k A^{\beta} \theta+m A^{\alpha} u_{t} & =0,
\end{aligned}
$$

with initial conditions

$$
u(0)=u_{0}, \quad u_{t}(0)=u_{1}, \quad \theta(0)=\theta_{0},
$$

with given constants $\rho, \sigma, c, k>0$ and $m \neq 0$. We are interested in describing the asymptotic behavior of solutions $u, \theta:[0, \infty) \rightarrow H$ as $t \rightarrow \infty$, in particulary describing the parameter regions for $(\alpha, \beta, \gamma)$ where exponential, non-exponential or polynomial stability is given.

The case $\gamma=0$ (equivalently: $\mu=0$ ) has been studied in detail in $[12,13]$ and the references therein, e.g. [4]. Here we add the feature of an abstract inertial term given by $\mu A^{\gamma} u_{t t}$. The case $\gamma=\beta=1 / 2$ and $\alpha \leq 3 / 4$ was discussed in [6].

Second, we look at the Cattaneo version of the abstract system which is given by

$$
\begin{aligned}
\rho_{1} u_{t t}(t)+\mu A^{\gamma} u_{t t}(t)+\sigma A u(t)-m A^{\alpha} \theta(t) & =0, \\
\rho_{2} \theta_{t}(t)+B_{1} q(t)+m A^{\alpha} u_{t}(t) & =0, \\
\tau q_{t}(t)+q(t)+B_{2} \theta(t) & =0,
\end{aligned}
$$

with the relaxation parameter $\tau>0$, and with densely defined, close operators $B_{1}, B_{2}$ satisfying

$$
-B_{1} B_{2}=\kappa A^{\beta} \text {. }
$$

\footnotetext{
${ }^{0}$ AMS subject classification: 35 B 40, 35 M 13, 35 Q 74, 74 F 05

Keywords and phrases: exponential stability, polynomial stability, Fourier law of heat conduction, Cattaneo law of heat conduction, inertial term, general parameter system
} 
Here, again $u, \theta:[0, \infty) \rightarrow H$, and $q:[0, \infty) \rightarrow(H)^{r}$ for some $r \in \mathbb{N}$. The operator $B_{2}$ maps its domain in $H$ into $(H)^{r}$,

$$
B_{2}: D\left(B_{2}\right) \subset H \rightarrow(H)^{r}
$$

and

$$
B_{1}: D\left(B_{1}\right) \subset(H)^{r} \rightarrow H .
$$

The relation (1.6) represents for $\tau>0$ the Cattaneo law of heat conduction. For $\tau=0$ we have Fourier's law, and we recover system (1.1), (1.2).

The thermoelastic plate model from Example 1 below, with the Cattaneo law, is contained choosing realizations of the divergence operator "div" for $B_{1}$, and of the gradient operator " $\nabla$ " for $B_{2}$, and $m=n$ in $\mathbb{R}^{n}$.

We will demonstrate that the change from Fourier to Cattaneo leads to a loss of exponential stability in most coupled systems, i.e. the early examples of Timoshenko or the classical thermoelastic plate are not exotic but rather common; the exotic one is, maybe, second-order thermoelasticity. Here one should also know that there are thermoelastic systems - with secondorder elasticity - which behave very much the same, no matter if $\tau=0$ or if $\tau>0$, see [28, 29]; this is true even on a quantitative level, see [14].

The abstract systems (1.1), (1.2) and (1.4)-(1.6) cover a variety of examples as there are (for $\tau=0$ see [25]):

Example 1. Taking $\alpha=\beta=\gamma=\frac{1}{2}$ we have the linear thermoelastic plate equation with inertial term in $H=L^{2}(\Omega)$ with domain $D\left(A^{\frac{1}{2}}\right)=H^{2}(\Omega) \cap H_{0}^{1}(\Omega), \Omega$ a bounded domain in $\mathbb{R}^{n}$, and $A^{\frac{1}{2}} v=-\Delta v$ :

$$
\begin{aligned}
u_{t t}-\mu \Delta u_{t t}+\Delta^{2} u-\Delta \theta & =0, \\
\theta_{t}-\Delta \theta+\Delta u_{t} & =0,
\end{aligned}
$$

where $u$ denotes the deflection of the plate and $\theta$ the temperature difference. For bounded domains and the Fourier model $\tau=0$, there are many results in particular on exponential stability, in particular for $\mu=0$, see for example $[2,15,17,18,19,20,22,24,25]$ For results for the Cauchy problem or in general exterior domains see for example [7, 8, 9, 24, 25].

The corresponding Cattaneo model with $\tau>0$

$$
\begin{aligned}
u_{t t}+\Delta^{2} u-\Delta \theta & =0 \\
\theta_{t}+\operatorname{div} q+\Delta u_{t} & =0 \\
\tau q_{t}+q+\nabla \theta & =0
\end{aligned}
$$

looses the property of exponential stability for $\mu=0$, see [27] (for $\mu>0$ the exponential stability is always given [10]).

The Cauchy problem $\left(\Omega=\mathbb{R}^{n}\right)$ has been discussed for $\tau \geq 0$ and $\mu \geq 0$ in [31].

We remark that nonlinear versions of these equations have been studied recently, for bounded domains see [21] with $\tau=0$ and $\mu=0$, and [16] for $\mu>0$. The Cauchy problem was investigated 
in [32].

Example 2. Taking $\alpha=\frac{1}{2}, \beta=0, \mu=0$ we get the equations of linear viscoelasticity of the following type:

$$
u_{t t}+2 A u-\int_{0}^{t} g(t-r) A u(r) d r=0
$$

with the exponential kernel

$$
g(r)=e^{-r} .
$$

Example 3. The equations of linear second-order thermoelasticity in one space dimension:

$$
\begin{aligned}
u_{t t}+u_{x x}+\theta_{x}= & 0, \\
\theta_{t}-\theta_{x x}+u_{t x}= & 0, \\
u=\theta_{x}=0 & \text { on } \partial \Omega,
\end{aligned}
$$

where $\Omega=(0,1)$. This system corresponds to the case $\alpha=\frac{1}{2}, \beta=1, \mu=0$ with respect to the analysis of the asymptotic behavior, (but not formally since the gradient is not the root of the Laplacean), cp. [25].

The classical model for pure heat conduction using Fourier's law for the relation between the heat flux and the gradient of the temperature qualitatively yields exponentially stable systems for bounded reference configurations. This kind of stability remains the same if one replaces Fourier's law by Cattaneo's (Maxwell's, Vernotte's, ... ) law. Considering thermal and, simultaneously, elastic effects, this similarity with respect to exponential stability remains the same for classical second-order thermoelastic systems, Example 3 above, one (Fourier) being a hyperbolicparabolic coupling, the other one (Cattaneo) being a fully hyperbolic system. The similarities even extend to the asymptotical behavior of solutions to corresponding nonlinear systems. But for thermoelastic plates, Example 1 above, the picture changes drastically, i.e., this thermoelastic system changes its behavior from an exponentially stable to a non-exponentially stable one, while changing Fourier's law to Cattaneo's law. This raises the question of the "right" modeling.

This effect is also known for Timoshenko beams. In models for beams of Timoshenko type, a given exponentially stability triggered by a typical memory (history) term, is preserved by adding heat conduction in form of the Fourier model, but is lost - hence "destroyed" - by the Cattaneo model. The four differential equations in the model are given by

$$
\begin{aligned}
\rho_{1} \varphi_{t t}-k\left(\varphi_{x}+\psi_{x}\right)_{x} & =0, \\
\rho_{2} \psi_{t t}-b \psi_{x x}+\int_{0}^{\infty} \mathrm{e}^{-s} \psi_{x x}(t-s, \cdot) d s+k\left(\varphi_{x}+\psi\right)+\delta \theta_{x} & =0, \\
\rho_{3} \theta_{t}+q_{x}+\delta \psi_{t x} & =0 \\
\tau q_{t}+q+\theta_{x} & =0 .
\end{aligned}
$$

Here, the functions $\phi$ and $\psi$ model the transverse displacement of a beam with reference configuration $(0,1)$ respectively the rotation angle of a filament. $\theta$ and $q$ denote again the temperature 
difference and the heat flux, respectively. The material constants $\rho_{1}, \rho_{2}, k, b, \delta, \rho_{3}$ are positive, as well as the relaxation parameter $\tau$. The last differential equation represents the Cattaneo law (and the Fourier law for $\tau=0$ ). The term $\int_{0}^{\infty} \mathrm{e}^{-s} \psi_{x x}(t-s, \cdot) d s$ models the additional consideration of the history.

Assuming the (academic, in general physically not satisfied) condition

$$
\frac{\rho_{1}}{k}=\frac{\rho_{2}}{b},
$$

which corresponds to the equality of the wave speeds for $\phi$ and $\psi$, we have the following picture:

For $\delta=0$, it is a hyperbolic system with history term for $(\phi, \psi)$, and exponential stability is given. For the coupled system with $\delta \neq 0$ and $\tau=0$ (Fourier), the exponential stability is preserved. But for $\delta \neq 0, \tau>0$ (Cattaneo), the exponential stability is lost [11]. Again the question of an appropriate modeling comes up.

The stability analysis below will be made first for the Fourier system (1.1), (1.2) using semigroup techniques.

Here, as well as for the Cattaneo system, the analysis of the well-posedness is a non-trivial issue. The difficulty arises from the point of regularity of solutions visible in the regularity of $u_{t}$ if $\alpha$ is large, or in that of $\theta$ if $\alpha$ is large compared to $\beta$. This problem is solved in the larger, most interesting part of the parameter region by factorizing the operators and defining the domains appropriately depending on $\alpha \leq 1 / 2$ or $\alpha>1 / 2$. The regularity properties have to be taken into account also in the proofs of (non-)exponential stability.

The non-exponential stability for Cattaneo systems will be proved in constructing slowly decaying solutions with the Hurwitz criterion. For this part we will assume that $A$ has a countable system of eigenfunctions $\left(\phi_{j}\right)_{j}$ with corresponding increasing eigenvalues $0<\lambda_{j} \rightarrow \infty$ as $j \rightarrow \infty$. This is, e.g., satisfied for Examples 1 given above in bounded domains.

Summarizing, we

- present a detailed analysis of the impact of the inertial term giving a precise description of parameter regions of (non-)exponential stability, and

- demonstrate that the change from Fourier to Cattaneo leads to a loss of exponential stability in "most" coupled systems.

The paper is organized as follows: In Section 2 we present well-posedness results and we describe the region of parameters, where exponential stability is given for the Fourier model (1.1), (1.2). The region of non-exponential stability is characterized in Section 3. The loss of exponential stability for the bigger part of the parameter regions is proved in Section 4, also including the well-posedness issue in Section 4.3. Section 5 provides the regions of polynomial stability within regions of non-exponential stability.

The symbols $(\cdot, \cdot)$ and $\|\cdot\|$ are used to denote the inner product and norm in $H$ or multiple copies $[H]^{r}$, respectively. 


\section{Exponential stability region for the Fourier law}

Here we look at the Fourier model (1.1), (1.2), and we will describe the region of parameters where exponential stability is given.

We obtain for a regular solution in multiplying (1.1) by $u_{t}$, and (1.2) by $\theta$ the equality

$$
\frac{1}{2} \frac{d}{d t}\left[\rho\left\|u_{t}\right\|^{2}+\mu\left\|A^{\gamma / 2} u_{t}\right\|^{2}+\sigma\left\|A^{1 / 2} u\right\|^{2}+c\|\theta\|^{2}\right]+k\left\|A^{\beta / 2} \theta\right\|^{2}=0
$$

This motivates the choice of the Hilbert space

$$
\mathcal{H}=D\left(A^{1 / 2}\right) \times D\left(A^{\gamma / 2}\right) \times H
$$

with inner product defined for $U_{j}=\left(u_{j}, v_{j}, \theta_{j}\right)^{T} \in \mathcal{H}, j=1,2$, as

$$
\left\langle U_{1}, U_{2}\right\rangle_{\mathcal{H}}=\sigma\left(A^{1 / 2} u_{1}, A^{1 / 2} u_{2}\right)+\mu\left(A^{\gamma / 2} v_{1}, A^{\gamma / 2} v_{2}\right)+\rho\left(v_{1}, v_{2}\right)+c\left(\theta_{1}, \theta_{2}\right) .
$$

Now, system (1.1)-(1.3) can be written as an evolution equation on $\mathcal{H}$ given by

$$
\left\{\begin{array}{l}
\frac{d U}{d t}=\mathcal{A}_{\alpha, \beta, \gamma} U, \\
U(0)=U_{0}
\end{array}\right.
$$

where $U_{0}=\left(u_{0}, v_{0}, \theta_{0}\right)^{T}$, and the operator $\mathcal{A}_{\alpha, \beta, \gamma}: D\left(\mathcal{A}_{\alpha, \beta, \gamma}\right) \subset \mathcal{H} \rightarrow \mathcal{H}$ is defined for $\underline{\alpha \leq 1 / 2}$ by

$$
\mathcal{A}_{\alpha, \beta, \gamma}\left(\begin{array}{c}
u \\
v \\
\theta
\end{array}\right)=\left(\begin{array}{c}
v \\
-\left(I+\frac{\mu}{\rho} A^{\gamma}\right)^{-1} A^{\alpha}\left\{\frac{\sigma}{\rho} A^{1-\alpha} u-\frac{m}{\rho} \theta\right\} \\
-\frac{m}{c} A^{\alpha} v-\frac{k}{c} A^{\beta} \theta
\end{array}\right)
$$

with domain

$D\left(\mathcal{A}_{\alpha, \beta, \gamma}\right)=\left\{(u, v, \theta)^{T} \in \mathcal{H} \mid v \in D\left(A^{1 / 2}\right), \theta \in D\left(A^{\beta}\right), u \in D\left(A^{1-\alpha}\right), \sigma A^{1-\alpha} u-m \theta \in D\left(A^{\alpha-\gamma / 2}\right)\right\}$.

For $\alpha>1 / 2$ it is defined by

$$
\mathcal{A}_{\alpha, \beta, \gamma}\left(\begin{array}{c}
u \\
v \\
\theta
\end{array}\right)=\left(\begin{array}{c}
v \\
-\left(I+\frac{\mu}{\rho} A^{\gamma}\right)^{-1} A^{\alpha}\left\{\frac{\sigma}{\rho} A^{1-\alpha} u-\frac{m}{\rho} \theta\right\} \\
-A^{\alpha-1 / 2}\left\{\frac{m}{c} A^{1 / 2} v+\frac{k}{c} A^{\beta-(\alpha-1 / 2)} \theta\right\}
\end{array}\right)
$$

with domain

$$
\begin{aligned}
D\left(\mathcal{A}_{\alpha, \beta, \gamma}\right)= & \left\{(u, v, \theta)^{T} \in \mathcal{H} \mid v \in D\left(A^{1 / 2}\right), \sigma A^{1-\alpha} u-m \theta \in D\left(A^{\alpha-\gamma / 2}\right),\right. \\
& \left.A^{\beta-(\alpha-1 / 2)} \theta \in H, A^{\alpha-1 / 2}\left[m A^{1 / 2} v+k A^{\beta-(\alpha-1 / 2)} \theta\right] \in H\right\} .
\end{aligned}
$$


Note that $\mathcal{A}_{\alpha, \beta, \gamma}$ is dissipative, cf. (2.1), and the density of $D\left(\mathcal{A}_{\alpha, \beta, \gamma}\right)$ in $\mathcal{H}$ is a consequence of the density of $D(A)$ in $H$. Therefore, it is sufficient to prove that $0 \in \rho\left(\mathcal{A}_{\alpha, \beta, \gamma}\right)$. We will do this in detail for the parameter region where

$$
\beta \geq 2 \alpha-1
$$

The region $\beta<2 \alpha-1$ (corresponding to region $S_{3}$ in Figure 2.1) is left open, cf. [1,5] for regions where 0 might not belong to the resolvent set. We now solve the problem

$$
\mathcal{A}_{\alpha, \beta, \gamma} U=F \equiv(f, g, h)^{T} \text { in } \mathcal{H} .
$$

First, let $\alpha \leq 1 / 2$ : Then, defining

$v:=f, \quad \theta:=-A^{-\beta}\left(\frac{c}{k} h+\frac{m}{k} A^{\alpha} f\right), \quad u:=A^{-(1-\alpha)}\left(\frac{m}{\sigma} \theta+\frac{1}{\sigma} A^{-(\alpha-\gamma / 2)}\left(\rho A^{-\gamma / 2} g+\mu A^{\gamma / 2} g\right)\right)$,

we have $(u, v, \theta)^{T} \in D\left(\mathcal{A}_{\alpha, \beta, \gamma}\right)$ and

$$
\begin{gathered}
\|v\|_{D\left(A^{\gamma / 2}\right)} \leq c_{1}\|f\|_{D\left(A^{1 / 2}\right)}, \quad\|\theta\| \leq c_{1}\left(\|h\|+\left\|A^{1 / 2} f\right\|,\right. \\
\|u\|_{D\left(A^{1 / 2}\right)} \leq c_{1}\left\|A^{-(1 / 2-\alpha)} \theta\right\|+c_{1}\left\|\rho A^{-\gamma / 2} g+\mu A^{\gamma / 2} g\right\| \leq c_{1}\left(\|\theta\|+\|g\|_{D\left(A^{\gamma / 2}\right)}\right),
\end{gathered}
$$

where we used $\alpha \leq 1 / 2$, and where $c_{1}$ denotes a generic constant. Hence we have $U \in D\left(\mathcal{A}_{\alpha, \beta, \gamma}\right)$, $\mathcal{A}_{\alpha, \beta, \gamma} U=F$ and $\|U\|_{\mathcal{H}} \leq c_{1}\|F\|_{\mathcal{H}}$.

Now, let $\alpha>1 / 2$ : Then, defining

$$
\begin{aligned}
v & :=f, \quad \theta:=-A^{-(\beta-(\alpha-1 / 2))}\left(\frac{c}{k} A^{-(\alpha-1 / 2)} h+\frac{m}{k} A^{1 / 2} f\right), \\
u & :=A^{-(1-\alpha)}\left(\frac{m}{\sigma} \theta+\frac{1}{\sigma} A^{-(\alpha-\gamma / 2)}\left(\rho A^{-\gamma / 2} g+\mu A^{\gamma / 2} g\right)\right),
\end{aligned}
$$

we have $(u, v, \theta)^{T} \in D\left(\mathcal{A}_{\alpha, \beta, \gamma}\right)$ and

$$
\|v\|_{D\left(A^{\gamma / 2}\right)} \leq c_{1}\|f\|_{D\left(A^{1 / 2}\right)}, \quad\|\theta\| \leq c_{1}\left(\left\|A^{\beta-(\alpha-1 / 2)} \theta\right\| \leq c_{1}\|F\|_{\mathcal{H}}\right.
$$

and the estimate for $u$ is obtained by

$$
\begin{aligned}
\|u\|_{D\left(A^{1 / 2}\right)} & \leq c_{1}\left\|A^{-(\beta-(2 \alpha-1))}\right\|_{H \rightarrow H}\left\|A^{\beta-(\alpha-1 / 2)} \theta\right\|+c_{1}\|F\|_{\mathcal{H}} \\
& \leq c_{1}\|F\|_{\mathcal{H}},
\end{aligned}
$$

where we used the assumption $\beta \geq 2 \alpha-1$. Hence we have again $U \in D\left(\mathcal{A}_{\alpha, \beta, \gamma}\right), \mathcal{A}_{\alpha, \beta, \gamma} U=F$ and $\|U\|_{\mathcal{H}} \leq c_{1}\|F\|_{\mathcal{H}}$.

Consequently, $\mathcal{A}_{\alpha, \beta, \gamma}$ generates a $C_{0}$-semigroup $\left\{e^{\mathcal{A}_{\alpha, \beta, \gamma} t}\right\}_{t \geq 0}$ of contractions on $\mathcal{H}$. Then $U$, given by

$$
U(t)=e^{\mathcal{A}_{\alpha, \beta, \gamma} t} U_{0}
$$

is the solution to (2.2) with

$$
U \in C^{1}([0, \infty) ; \mathcal{H}) \cap C^{0}\left([0, \infty) ; D\left(\mathcal{A}_{\alpha, \beta, \gamma}\right)\right) .
$$


Remark 2.1. In order to simplify our notation, let $V_{\delta}$ denote the space $D\left(A^{\delta}\right)$. For example, using this notation, the Hilbert space $\mathcal{H}$ is given by

$$
\mathcal{H}=V_{1 / 2} \times V_{\gamma / 2} \times H
$$

In order to prove the exponential stability, it is useful to recall the following known result (see for example [26]):

Theorem 2.2. Let $\left\{e^{A t}\right\}_{t \geq 0}$ be a $C_{0}$-semigroup of contractions on a Hilbert space $H$. Then the semigroup is exponentially stable if and only if $i \mathbb{R} \in \rho(A)$ (resolvent set) and

$$
\limsup _{\lambda \in \mathbb{R},|\lambda| \rightarrow \infty}\left\|(i \lambda I-A)^{-1}\right\|_{\mathcal{L}(H)}<\infty .
$$

It will allow us to prove

Theorem 2.3. Let $\alpha, \beta, \gamma \in S$ where $S$ is given by

$$
S=\left\{(\alpha, \beta) \in[0,1] \times[0,1], 0<\gamma \leq 1: \frac{1}{2} \leq \alpha+\frac{\beta}{2}, \quad \frac{\gamma}{2} \leq \alpha-\frac{\beta}{2} \leq \frac{1}{2}\right\} .
$$

Then the semigroup $\left\{e^{\mathcal{A}_{\alpha, \beta, \gamma} t}\right\}_{t \geq 0}$ is exponentially stable.

In Section 3 we will demonstrate the optimality of this result in the sense that for parameters outside the set $S$, the non-exponential stability will be proved.

A typical region $S$ is given for small positive $\gamma$ by Figure 2.1

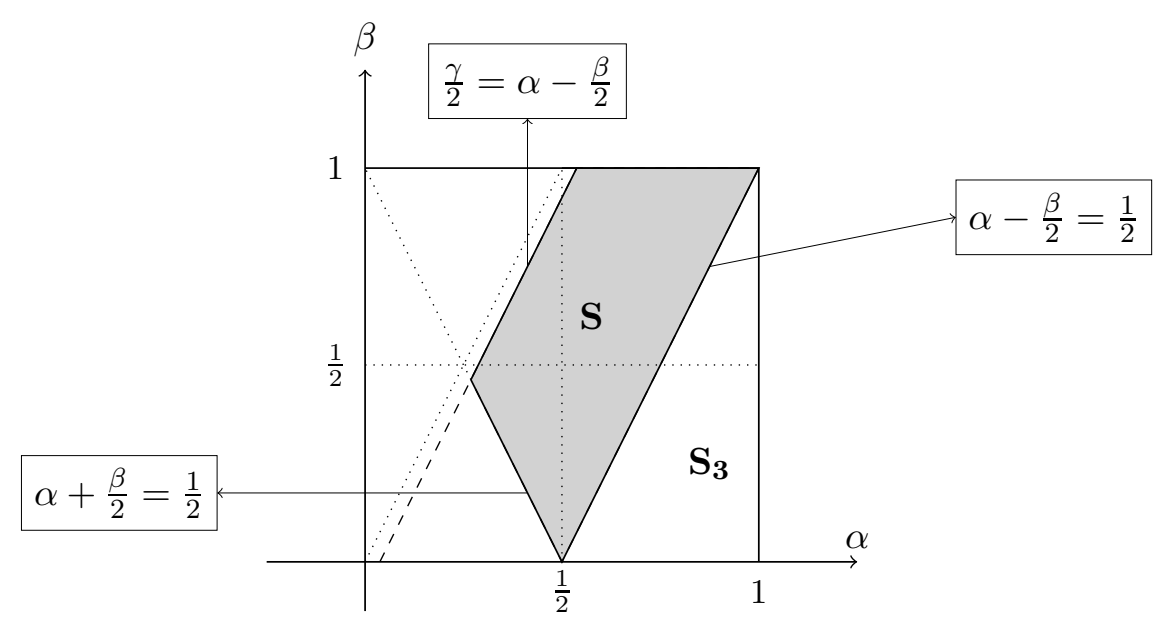

Figure 2.1: Region $S$.

Proof. We prove condition (2.12) considering the resolvent equation

$$
i \lambda U-\mathcal{A}_{\alpha, \beta, \gamma} U=F, \quad \text { in } \quad \mathcal{H}
$$


where $U=(u, v, \theta)^{T}$ and $F=(f, g, h)^{T}$, we have:

$$
\begin{aligned}
& i \lambda u-v=f, \quad \text { in } \quad V_{1 / 2}, \\
& i \lambda v+\left(I+\frac{\mu}{\rho} A^{\gamma}\right)^{-1} A^{\alpha}\left(\frac{\sigma}{\rho} A^{1-\alpha} u-\frac{m}{\rho} \theta\right)=g, \quad \text { in } \quad V_{\gamma / 2}, \\
& i \lambda \theta+\frac{T_{1}}{c}=h, \quad \text { in } \quad H,
\end{aligned}
$$

where

$$
T_{1}:= \begin{cases}k A^{\beta} \theta+m c A^{\alpha} v, & \text { for } \alpha \leq 1 / 2, \\ A^{\alpha-1 / 2}\left(m A^{1 / 2} v+k A^{\beta-(\alpha-1 / 2)} \theta\right), & \text { for } \alpha>1 / 2 .\end{cases}
$$

Now, using $v \in V_{1 / 2} \subset V_{\gamma / 2}$ and $\left(I+\frac{\mu}{\rho} A^{\gamma}\right): V_{\gamma / 2} \rightarrow V_{\gamma / 2}^{\prime}$ being an isomorphism, we have

$$
\begin{aligned}
& i \lambda u-v=f, \quad \text { in } \quad V_{1 / 2}, \\
& i \lambda \rho v+i \lambda \mu A^{\gamma} v+A^{\alpha}\left(\sigma A^{1-\alpha} u-m \theta\right)=\rho g+\mu A^{\gamma} g, \quad \text { in } \quad V_{\gamma / 2}^{\prime}, \\
& i \lambda c \theta+T_{1}=c h, \quad \text { in } \quad H .
\end{aligned}
$$

Now, multiplying the resolvent equation (2.14) by $U \in D\left(\mathcal{A}_{\alpha, \beta, \gamma}\right) \subset \mathcal{H}$, we deduce

$$
-\operatorname{Re}\left(\mathcal{A}_{\alpha, \beta, \gamma} U, U\right)_{\mathcal{H}}=\operatorname{Re}(U, F)_{\mathcal{H}}
$$

where

$$
-\left(\mathcal{A}_{\alpha, \beta, \gamma} U, U\right)_{\mathcal{H}}=-\sigma\left(A^{1 / 2} v, A^{1 / 2} u\right)+\left\langle A^{\alpha}\left(\sigma A^{1-\alpha} u-m \theta\right), v\right\rangle_{V_{\gamma / 2}^{\prime} \times V_{\gamma / 2}}+\left(T_{1}, \theta\right) .
$$

Here, by the definition of $D\left(\mathcal{A}_{\alpha, \beta, \gamma}\right)$ for $\alpha \leq 1 / 2$, we have $u \in V_{1-\alpha}, v \in V_{1 / 2} \subset V_{\alpha}$, and

$$
\left(\sigma A^{1-\alpha} u-m \theta\right) \in V_{\alpha-\gamma / 2} \subset H, \quad \text { because } \quad \alpha \geq \frac{\gamma}{2} \quad \text { in } \quad S .
$$

So we obtain for $\alpha \leq 1 / 2$

$$
\begin{aligned}
-\left(\mathcal{A}_{\alpha, \beta, \gamma} U, U\right)_{\mathcal{H}} & =-\left(A^{\alpha} v, \sigma A^{1-\alpha} u-m \theta\right)+\left\langle A^{\alpha}\left(\sigma A^{1-\alpha} u-m \theta\right), v\right\rangle_{V_{\gamma / 2}^{\prime} \times V_{\gamma / 2}}+k\left\|A^{\beta / 2} \theta\right\|^{2} \\
& =-\left(A^{\alpha} v, \sigma A^{1-\alpha} u-m \theta\right)+\left(\sigma A^{1-\alpha} u-m \theta, A^{\alpha} v\right)+k\left\|A^{\beta / 2} \theta\right\|^{2}
\end{aligned}
$$

which implies

$$
-\operatorname{Re}\left(\mathcal{A}_{\alpha, \beta, \gamma} U, U\right)_{\mathcal{H}}=k\left\|A^{\beta / 2} \theta\right\|^{2}, \quad \text { for } \quad \alpha \leq \frac{1}{2} .
$$

On the other hand, for $\alpha>1 / 2$, we have

$$
m A^{1 / 2} v+k A^{\beta-(\alpha-1 / 2)} \theta \in V_{\alpha-1 / 2} \subset H
$$

and

$$
\theta \in V_{\beta-(\alpha-1 / 2)} \subset V_{\beta-1 / 2} \subset V_{\alpha-1 / 2} \subset H \text {, because } \quad \alpha-\frac{1}{2} \leq \frac{\beta}{2} \quad \text { in } \quad S \text {. }
$$


Then we have

$$
\begin{aligned}
-\left(\mathcal{A}_{\alpha, \beta, \gamma} U, U\right)_{\mathcal{H}}= & -\sigma\left(A^{1 / 2} v, A^{1 / 2} u\right)+\left\langle A^{\alpha}\left(\sigma A^{1-\alpha} u-m \theta\right), v\right\rangle_{V_{\gamma / 2}^{\prime} \times V_{\gamma / 2}} \\
& +\left(m A^{1 / 2} v+k A^{\beta-(\alpha-1 / 2)} \theta, A^{\alpha-1 / 2} \theta\right) \\
= & -\left(A^{1 / 2} v, A^{\alpha-1 / 2}\left(\sigma A^{1-\alpha} u-m \theta\right)\right)+\left(A^{\alpha-1 / 2}\left(\sigma A^{1-\alpha} u-m \theta\right), A^{1 / 2} v\right) \\
& +k\left\|A^{\beta / 2} \theta\right\|^{2},
\end{aligned}
$$

which implies

$$
-\operatorname{Re}\left(\mathcal{A}_{\alpha, \beta, \gamma} U, U\right)_{\mathcal{H}}=k\left\|A^{\beta / 2} \theta\right\|^{2}, \quad \text { for } \quad \alpha>\frac{1}{2} .
$$

Then, using (2.20)-(2.21) in (2.19) we obtain the first inequality (corresponding to (2.1))

$$
k\left\|A^{\beta / 2} \theta\right\|^{2} \leq C\|U\|_{\mathcal{H}}\|F\|_{\mathcal{H}} .
$$

Here and in the sequel $C, C_{1}, \ldots$ will denote positive constants, in particular being independent of $\lambda$.

Now, using equation (2.17) in $u \in V_{1 / 2} \hookrightarrow V_{\gamma / 2}, 0<\gamma \leq 1$, we have

$$
\left\langle i \lambda \rho v+i \lambda \mu A^{\gamma} v, u\right\rangle_{V_{\gamma / 2}^{\prime} \times V_{\gamma / 2}}+\left\langle A^{\alpha}\left(\sigma A^{1-\alpha} u-m \theta\right), v\right\rangle_{V_{\gamma / 2}^{\prime} \times V_{\gamma / 2}}=\rho\left\langle g+\mu A^{\gamma} g, u\right\rangle_{V_{\gamma / 2}^{\prime} \times V_{\gamma / 2}} .
$$

Then, using equation (2.16), we have

$$
\left\langle\rho v+\mu A^{\gamma} v,-v-f\right\rangle_{V_{\gamma / 2}^{\prime} \times V_{\gamma / 2}}+\left\langle A^{\alpha}\left(\sigma A^{1-\alpha} u-m \theta\right), v\right\rangle_{V_{\gamma / 2}^{\prime} \times V_{\gamma / 2}}=\rho\left\langle g+\mu A^{\gamma} g, u\right\rangle_{V_{\gamma / 2}^{\prime} \times V_{\gamma / 2}}
$$

which implies, for $v \in V_{1 / 2} \hookrightarrow V_{\gamma / 2}$,

$$
\begin{aligned}
\underbrace{\left\langle A^{\alpha}\left(\sigma A^{1-\alpha} u-m \theta\right), u\right\rangle_{V_{\gamma / 2}^{\prime} \times V_{\gamma / 2}}=}_{=: I_{3}} & \rho(v, f)+\mu\left\|A^{\gamma / 2} v\right\|^{2}+\rho\|v\|^{2}+\underbrace{\mu\left\langle A^{\gamma} v, f\right\rangle_{V_{\gamma / 2}^{\prime} \times V_{\gamma / 2}}}_{=: I_{1}} \\
& +\underbrace{\rho\left\langle g+\mu A^{\gamma} g, u\right\rangle_{V_{\gamma / 2}^{\prime} \times V_{\gamma / 2}}}_{=: I_{2}} .
\end{aligned}
$$

Note that

$$
\left|I_{1}\right|=\mu\left|\left(A^{\gamma / 2} v, A^{\gamma / 2} f\right)\right| \leq C\left\|A^{\gamma / 2} v\right\|\left\|A^{\gamma / 2} f\right\| \leq C_{1}\left\|A^{\gamma / 2} v\right\|\left\|A^{1 / 2} f\right\| \leq C\|U\|_{\mathcal{H}}\|F\|_{\mathcal{H}},
$$

because $V_{1 / 2} \hookrightarrow V_{\gamma / 2}$. Similarly we have

$$
\begin{aligned}
\left|I_{2}\right| \leq \rho|(g, u)|+\rho \mu\left|\left(A^{\gamma / 2} g, A^{\gamma / 2} u\right)\right| & \leq C\|U\|_{\mathcal{H}}\|F\|_{\mathcal{H}}+C\left\|A^{\gamma / 2} g\right\|\left\|A^{\gamma / 2} u\right\| \\
& \leq C\|U\|_{\mathcal{H}}\|F\|_{\mathcal{H}}+C_{1}\left\|A^{\gamma / 2} g\right\|\left\|A^{1 / 2} u\right\| \\
& \leq C\|U\|_{\mathcal{H}}\|F\|_{\mathcal{H}} .
\end{aligned}
$$

For the term $I_{3}$, in the case $\alpha \leq 1 / 2$ we have $u \in V_{1 / 2} \subset V_{\alpha}$ and $u \in V_{1-\alpha}$. Consequently

$$
I_{3}=\left(\sigma A^{1-\alpha} u-m \theta, A^{\alpha} u\right)=\sigma\left(A^{1-\alpha} u, A^{\alpha} u\right)-m\left(\theta, A^{\alpha} u\right) .
$$


So, using that $0 \leq \alpha-\frac{\beta}{2} \leq \frac{1}{2}$ in $S$, we deduce

$$
I_{3}=\sigma\left\|A^{1 / 2} u\right\|^{2}-m\left(A^{\beta / 2} \theta, A^{\alpha-\beta / 2} u\right), \quad \text { for } \quad \alpha \leq \frac{1}{2} .
$$

In the case $\alpha>1 / 2$, using that $0<\alpha-\frac{1}{2} \leq \beta$ in $S$, we obtain

$$
I_{3}=\left(A^{\alpha-1 / 2}\left(\sigma A^{1-\alpha} u-m \theta\right), A^{1 / 2} u\right)=\sigma\left\|A^{1 / 2} u\right\|^{2}-m\left(A^{\alpha-1 / 2} \theta, A^{1 / 2} u\right)
$$

Therefore, using (2.24)-(2.27) in (2.23) we obtain

$$
\sigma\left\|A^{1 / 2} u\right\|^{2} \leq C\|U\|_{\mathcal{H}}\|F\|_{\mathcal{H}}+\rho\|v\|^{2}+\mu\left\|A^{\gamma / 2} v\right\|^{2}+m\left|T_{2}\right|
$$

where

$$
T_{2}:= \begin{cases}\left(A^{\beta / 2} \theta, A^{\alpha-\beta / 2} u\right), & \text { for } \quad \alpha \leq 1 / 2, \\ \left(A^{\alpha-1 / 2} \theta, A^{1 / 2} u\right), & \text { for } \quad \alpha>1 / 2 .\end{cases}
$$

Note that, using the definition of $S$, we have $0 \leq \alpha-\frac{\beta}{2} \leq \frac{1}{2}$ for $\alpha \leq 1 / 2$, and $0<\alpha-\frac{1}{2} \leq \frac{\beta}{2}$ for $\alpha>1 / 2$, which implies

$$
\left|T_{2}\right| \leq C\left\|A^{\beta / 2} \theta\right\|\left\|A^{1 / 2} u\right\|, \quad \text { for all } \quad 0 \leq \alpha \leq 1 .
$$

Therefore, from (2.28), using (2.22), we have

$$
\sigma\left\|A^{1 / 2} u\right\|^{2} \leq C\|U\|_{\mathcal{H}}\|F\|_{\mathcal{H}}+C_{1}\left[\rho\|v\|^{2}+\mu\left\|A^{\gamma / 2} v\right\|^{2}\right] .
$$

Now, let us define further multipliers given by $\psi$ and $\phi$ as the solutions to the equations

$$
A^{\alpha} \psi=v \quad \text { and } \quad A^{\alpha} \phi=\theta .
$$

Since $\theta \in V_{\beta / 2}$ and $\frac{\gamma}{2} \leq \alpha+\frac{\beta}{2}$ in $S$, we have the regularity $\phi \in V_{\alpha+\beta / 2} \hookrightarrow V_{\gamma / 2}$. Then, with equation (2.17), we have for $\phi \in V_{\gamma / 2}$

$$
\left\langle i \lambda \rho v+i \lambda \mu A^{\gamma} v+A^{\alpha}\left(\sigma A^{1-\alpha} u-m \theta\right), \phi\right\rangle_{V_{\gamma / 2}^{\prime} \times V_{\gamma / 2}}=\left\langle\rho g+\mu A^{\gamma} g, \phi\right\rangle_{V_{\gamma / 2}^{\prime} \times V_{\gamma / 2}},
$$

or

$$
\begin{aligned}
\underbrace{i \lambda \rho(v, \phi)}_{=: I_{4}}+ & \underbrace{i \lambda \mu\left\langle A^{\gamma} v, \phi\right\rangle_{V_{\gamma / 2}^{\prime} \times V_{\gamma / 2}}}_{=: I_{5}}+ \\
& \underbrace{\left\langle A^{\alpha}\left(\sigma A^{1-\alpha} u-m \theta\right), \phi\right\rangle_{V_{\gamma / 2}^{\prime} \times V_{\gamma / 2}}}_{=: I_{6}}=\rho(g, \phi)+\mu\left\langle A^{\gamma} g, \phi\right\rangle_{V_{\gamma / 2}^{\prime} \times V_{\gamma / 2}} .
\end{aligned}
$$

Using (2.30) and equation (2.18), we get

$$
\begin{aligned}
I_{4} & =i \lambda \rho\left(A^{\alpha} \psi, \phi\right)=i \lambda \rho\left(\psi, A^{\alpha} \phi\right) \\
& =i \lambda \rho(\psi, \theta)=\rho(\psi,-i \lambda \theta) \\
& =\rho\left(\psi, \frac{1}{c} T_{1}-h\right),
\end{aligned}
$$


where $T_{1}$ was defined in (2.15). Here, for $\alpha \leq 1 / 2$ we have $v \in V_{1 / 2} \subset V_{\alpha}$. Then

$$
\begin{aligned}
I_{4} & =\rho\left(\psi, \frac{k}{c} A^{\beta} \theta+\frac{m}{c} A^{\alpha} v-h\right) \\
& =\frac{k \rho}{c}\left(\psi, A^{\beta} \theta\right)+\frac{m \rho}{c}\|v\|^{2}-\rho(\psi, h), \quad \text { for } \quad \alpha \leq \frac{1}{2} .
\end{aligned}
$$

Similarly, for $\alpha>1 / 2$, from (2.30) we have $\psi \in V_{1 / 2+\alpha} \subset V_{\alpha-1 / 2} \subset H$. Then, using equations (2.18)-(2.15) ${ }_{2}$, we get again

$$
\begin{aligned}
I_{4} & =\rho\left(\psi, A^{\alpha-1 / 2}\left(\frac{m}{c} A^{1 / 2} v+\frac{k}{c} A^{\beta-(\alpha-1 / 2)} \theta\right)-h\right) \\
& =\rho\left(A^{\alpha-1 / 2} \psi, \frac{m}{c} A^{1 / 2} v+\frac{k}{c} A^{\beta-(\alpha-1 / 2)} \theta\right)-\rho(\psi, h) \\
& =\frac{k \rho}{c}\left(\psi, A^{\beta} \theta\right)+\frac{m \rho}{c}\|v\|^{2}-\rho(\psi, h), \quad \text { for } \quad \alpha>\frac{1}{2} .
\end{aligned}
$$

For $I_{5}$, using $v \in V_{1 / 2} \subset V_{\gamma / 2}$ and $\theta \in V_{\beta-(\alpha-1 / 2)}$ we have

$$
A^{\gamma-\alpha} v \in V_{\alpha-\gamma / 2} \subset H \quad \text { and } \quad A^{\alpha} \phi=\theta \in V_{\beta-(\alpha-1 / 2)} \subset V_{\alpha-\gamma / 2}^{\prime} .
$$

So we can identify

$$
\begin{aligned}
I_{5} & =i \lambda \mu\left\langle A^{\gamma-\alpha} v, A^{\alpha} \phi\right\rangle_{V_{\alpha-\gamma / 2} \times V_{\alpha-\gamma / 2}^{\prime}}=\mu\left\langle A^{\gamma-\alpha} v,-i \lambda \theta\right\rangle_{V_{\alpha-\gamma / 2} \times V_{\alpha-\gamma / 2}^{\prime}} \\
& =\frac{\mu}{c}\left\langle A^{\gamma-\alpha} v, T_{1}-c h\right\rangle_{V_{\alpha-\gamma / 2} \times V_{\alpha-\gamma / 2}^{\prime}},
\end{aligned}
$$

which implies, for $\alpha \leq 1 / 2$, that

$$
\begin{aligned}
I_{5} & =\frac{\mu}{c}\left\langle A^{\gamma-\alpha} v, k A^{\beta} \theta+m A^{\alpha} v-c h\right\rangle_{V_{\alpha-\gamma / 2} \times V_{\alpha-\gamma / 2}^{\prime}}, \\
& =\frac{\mu k}{c}\left\langle A^{\gamma-\alpha} v, A^{\beta} \theta\right\rangle_{V_{\alpha-\gamma / 2} \times V_{\alpha-\gamma / 2}^{\prime}}+\frac{\mu m}{c}\left\|A^{\gamma / 2} v\right\|^{2}-\mu\left\langle A^{\gamma-\alpha} v, h\right\rangle_{V_{\alpha-\gamma / 2} \times V_{\alpha-\gamma / 2}^{\prime}} .
\end{aligned}
$$

Similarly, for $\alpha>1 / 2$, using that

$$
A^{\alpha} v \in V_{\alpha-1 / 2}^{\prime} \subset V_{\alpha-\gamma / 2}^{\prime} \text { and } \theta \in V_{\beta-(\alpha-1 / 2)} \subset H,
$$

we obtain again

$$
\begin{aligned}
I_{5} & =\frac{\mu}{c}\left\langle A^{\gamma-\alpha} v, A^{\alpha-1 / 2}\left(m A^{1 / 2} v+k A^{\beta-(\alpha-1 / 2)} \theta\right)-c h\right\rangle_{V_{\alpha-\gamma / 2} \times V_{\alpha-\gamma / 2}^{\prime}} \\
& =\frac{\mu m}{c}\left\langle A^{\gamma-\alpha} v, A^{\alpha} v\right\rangle_{V_{\alpha-\gamma / 2} \times V_{\alpha-\gamma / 2}^{\prime}}+\frac{\mu k}{c}\left\langle A^{\gamma-\alpha} v, A^{\beta} \theta\right\rangle_{V_{\alpha-\gamma / 2} \times V_{\alpha-\gamma / 2}^{\prime}}-\mu\left\langle A^{\gamma-\alpha} v, h\right\rangle_{V_{\alpha-\gamma / 2} \times V_{\alpha-\gamma / 2}^{\prime}} \\
& =\frac{\mu k}{c}\left\langle A^{\gamma-\alpha} v, A^{\beta} \theta\right\rangle_{V_{\alpha-\gamma / 2} \times V_{\alpha-\gamma / 2}^{\prime}}+\frac{\mu m}{c}\left\|A^{\gamma / 2} v\right\|^{2}-\mu\left\langle A^{\gamma-\alpha} v, h\right\rangle_{V_{\alpha-\gamma / 2} \times V_{\alpha-\gamma / 2}^{\prime}}
\end{aligned}
$$

Finally, using that $\alpha \geq \frac{\gamma}{2}$ in $S$, we have $\left(\sigma A^{1-\alpha} u-m \theta\right) \in V_{\alpha-\gamma / 2} \subset H$. Then we have

$$
I_{6}=\left(\sigma A^{1-\alpha} u-m \theta, A^{\alpha} \phi\right)=\sigma\left(A^{1-\alpha} u, \theta\right)-m\|\theta\|^{2},
$$


where the last equality is valid because $u \in V_{1-\alpha}$.

Therefore, inserting (2.32)-(2.36) into (2.31) we deduce

$$
\begin{aligned}
\rho\|v\|^{2}+\mu\left\|A^{\gamma / 2} v\right\|^{2}= & c\|\theta\|^{2}-\frac{k \rho}{m}\left(A^{\alpha-1 / 2} \psi, A^{\beta-(\alpha-1 / 2)} \theta\right)-\frac{\mu k}{m}\left\langle A^{\gamma-\alpha} v, A^{\beta} \theta\right\rangle_{V_{\alpha-\gamma / 2} \times V_{\alpha-\gamma / 2}^{\prime}} \\
& -\frac{\sigma c}{m}\left(A^{1-\alpha} u, \theta\right)+\frac{\rho c}{m}(\psi, h)+\frac{\mu c}{m}\left\langle A^{\gamma-\alpha} v, h\right\rangle_{V_{\alpha-\gamma / 2} \times V_{\alpha-\gamma / 2}^{\prime}} \\
& +\frac{\rho c}{m}(g, \phi)+\frac{\mu c}{m}\left\langle A^{\gamma} g, \phi\right\rangle_{V_{\gamma / 2}^{\prime} \times V_{\gamma / 2}}
\end{aligned}
$$

Finally we will analyze the remaining terms in equation (2.37). In fact, in $S$ we have $\beta \leq 2 \alpha$, or $\beta-\alpha \leq \frac{\beta}{2}$, which implies, using (2.30) again,

$$
\begin{aligned}
\left|\left(A^{\alpha-1 / 2} \psi, A^{\beta-(\alpha-1 / 2)} \theta\right)\right| & =\left|\left(A^{\alpha} \psi, A^{\beta-\alpha} \theta\right)\right|=\left|\left(v, A^{\beta-\alpha} \theta\right)\right| \\
& \leq\left\|A^{\beta-\alpha} \theta\right\|\|v\| \leq C\left\|A^{\beta / 2} \theta\right\|\|v\| .
\end{aligned}
$$

Since in $S$ we have $\alpha+\frac{\beta}{2} \geq \frac{1}{2}$, we get

$$
\begin{aligned}
\left|\left(A^{1-\alpha} u, \theta\right)\right| & =\left|\left(A^{1-\alpha-\beta / 2} u, A^{\beta / 2} \theta\right)\right| \leq\left\|A^{1-\alpha-\beta / 2} u\right\|\left\|A^{\beta / 2} \theta\right\| \\
& \leq C_{1}\left\|A^{1 / 2} u\right\|\left\|A^{\beta / 2} \theta\right\| .
\end{aligned}
$$

Also by (2.13) we have

$$
\frac{\gamma}{2} \leq \alpha-\frac{\beta}{2} \Longleftrightarrow \gamma-\alpha+\frac{\beta}{2} \leq \frac{\gamma}{2}
$$

implying

$$
\begin{aligned}
\left|\left\langle A^{\gamma-\alpha} v, A^{\beta} \theta\right\rangle_{V_{\alpha-\gamma / 2} \times V_{\alpha-\gamma / 2}^{\prime}}\right| & =\left|\left(A^{\gamma-\alpha+\beta / 2} v, A^{\beta / 2} \theta\right)\right| \\
& \leq\left\|A^{\gamma-\alpha+\beta / 2} v\right\|\left\|A^{\beta / 2} \theta\right\| \leq C_{1}\left\|A^{\gamma / 2} v\right\|\left\|A^{\beta / 2} \theta\right\| .
\end{aligned}
$$

Now, using $\frac{\gamma}{2} \leq \alpha$, we have

$$
\left|\left\langle A^{\gamma-\alpha} v, h\right\rangle_{V_{\alpha-\gamma / 2} \times V_{\alpha-\gamma / 2}^{\prime}}\right| \leq C_{1}\left\|A^{\gamma-\alpha} v\right\|\|h\| \leq C_{1}\left\|A^{\gamma / 2} v\right\|\|h\| \leq C_{1}\|U\|_{\mathcal{H}}\|F\|_{\mathcal{H}} .
$$

Also we get

$$
|(\psi, h)| \leq\|\psi\|\|h\| \leq C_{1}\left\|A^{\alpha} \psi\right\|\|h\|=C_{1}\|v\|\|h\| \leq C_{1}\|U\|_{\mathcal{H}}\|F\|_{\mathcal{H}},
$$

and

$$
|(g, \phi)| \leq C_{1}\left\|A^{\gamma / 2} g\right\|\left\|A^{\alpha / 2} \phi\right\| \leq C_{1}\left\|A^{\gamma / 2} g\right\|\|\theta\| \leq C_{1}\|U\|_{\mathcal{H}}\|F\|_{\mathcal{H}} .
$$

Finally, using again $\frac{\gamma}{2} \leq \alpha$ and (2.30) we obtain

$$
\begin{aligned}
\left|\left\langle A^{\gamma} g, \phi\right\rangle_{V_{\gamma / 2}^{\prime} \times V_{\gamma / 2}}\right| & =\left|\left(A^{\gamma-\alpha} g, A^{\alpha} \phi\right)\right|=\left|\left(A^{\gamma-\alpha} g, \theta\right)\right| \\
& \leq C_{1}\left\|A^{\gamma / 2} g\right\|\|\theta\| \leq C_{1}\|U\|_{\mathcal{H}}\|F\|_{\mathcal{H}} .
\end{aligned}
$$


Therefore, using the inequalities (2.38)-(2.44) in (2.37) and using (2.22) we deduce

$$
\rho\|v\|^{2}+\mu\left\|A^{\gamma / 2} v\right\|^{2} \leq C_{1}\|U\|_{\mathcal{H}}\|F\|_{\mathcal{H}}+C_{1}\left\|A^{\beta / 2} \theta\right\|\left\|A^{1 / 2} u\right\| .
$$

Combining (2.22), (2.29), (2.45) we obtain

$$
\|U\|_{\mathcal{H}} \leq C\|F\|_{\mathcal{H}}
$$

which proves Theorem 2.3.

Remark 2.4. Let us give some examples of S-regions.

Example a.

$$
\gamma=\frac{1}{4}
$$

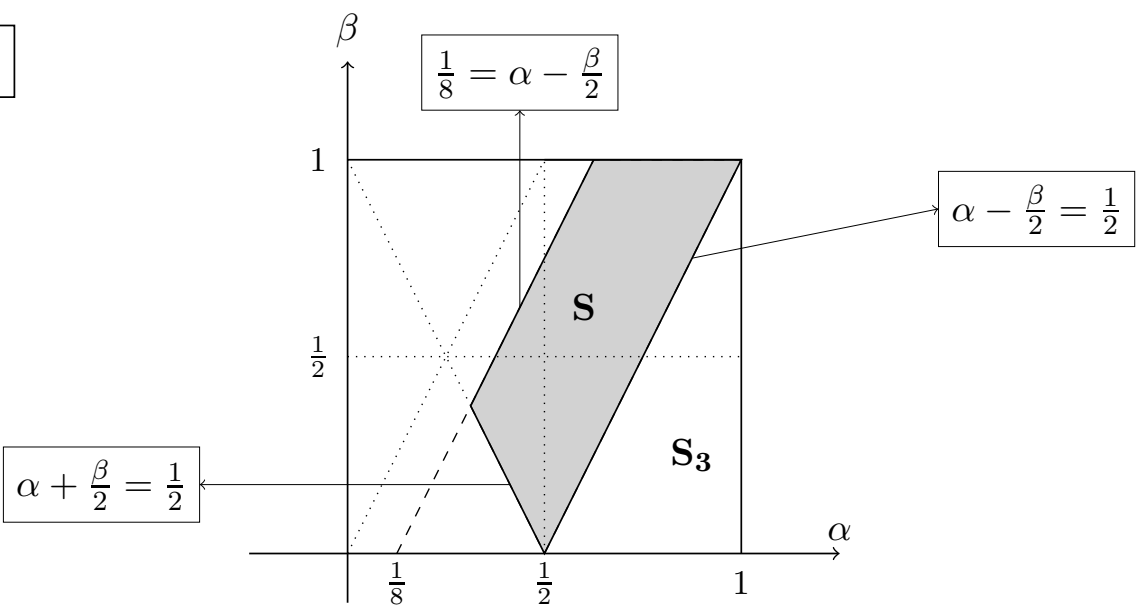

$$
\gamma=\frac{1}{2}
$$

Example b.

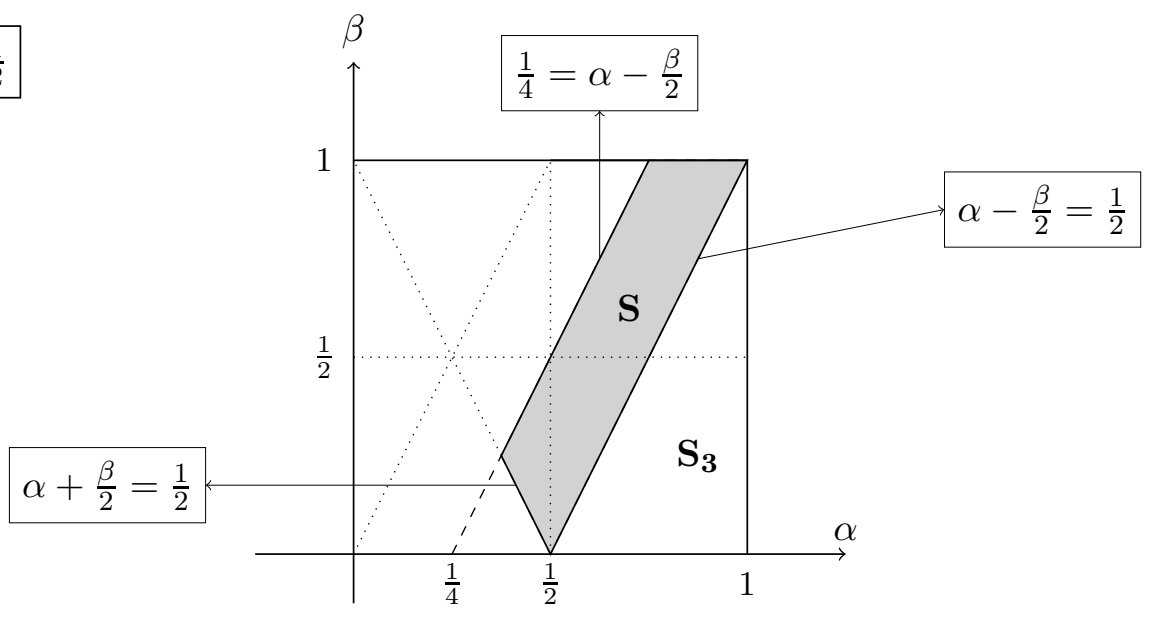




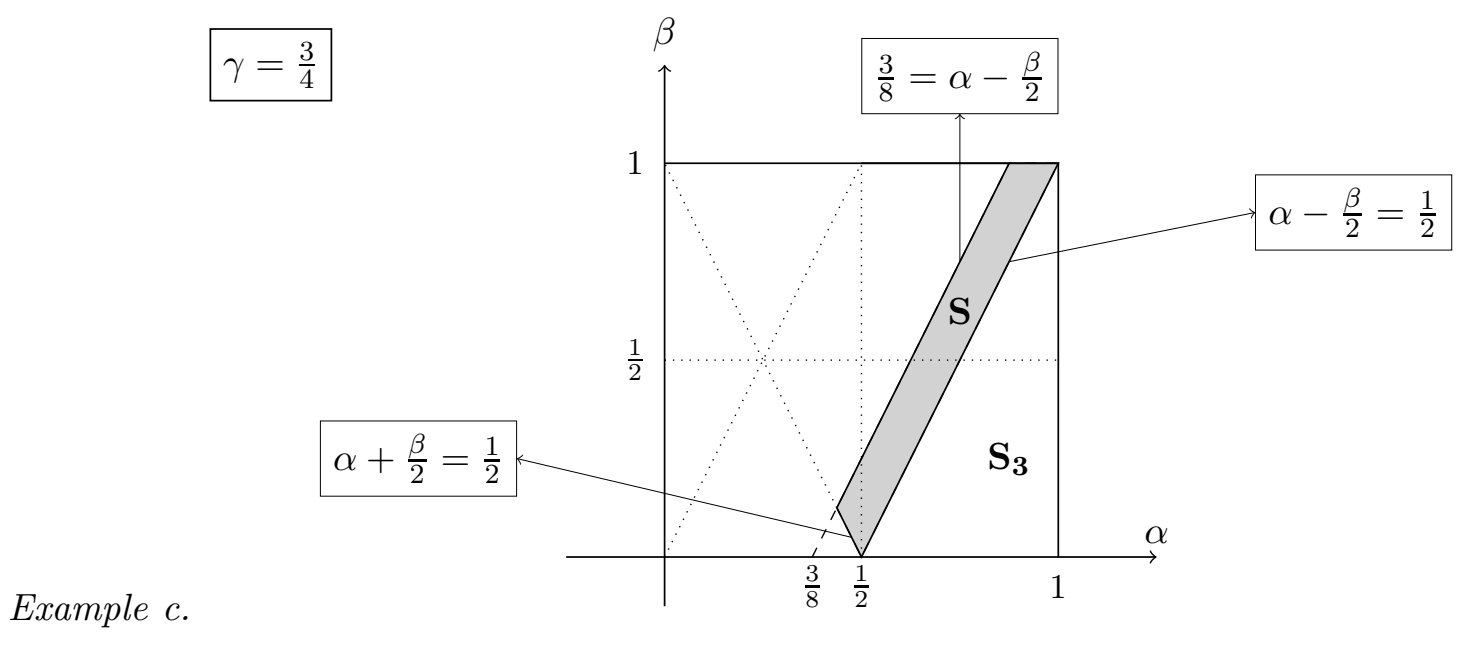

\section{Region of non-exponential stability for the Fourier law}

In order to complete the result given in Theorem 2.3 and to demonstrate its optimality, we will prove that the region of non-exponential stability is the complement of $S$, that is $S_{2} \cup S_{3}$ (see next figure). In fact, without loss of generality we assume

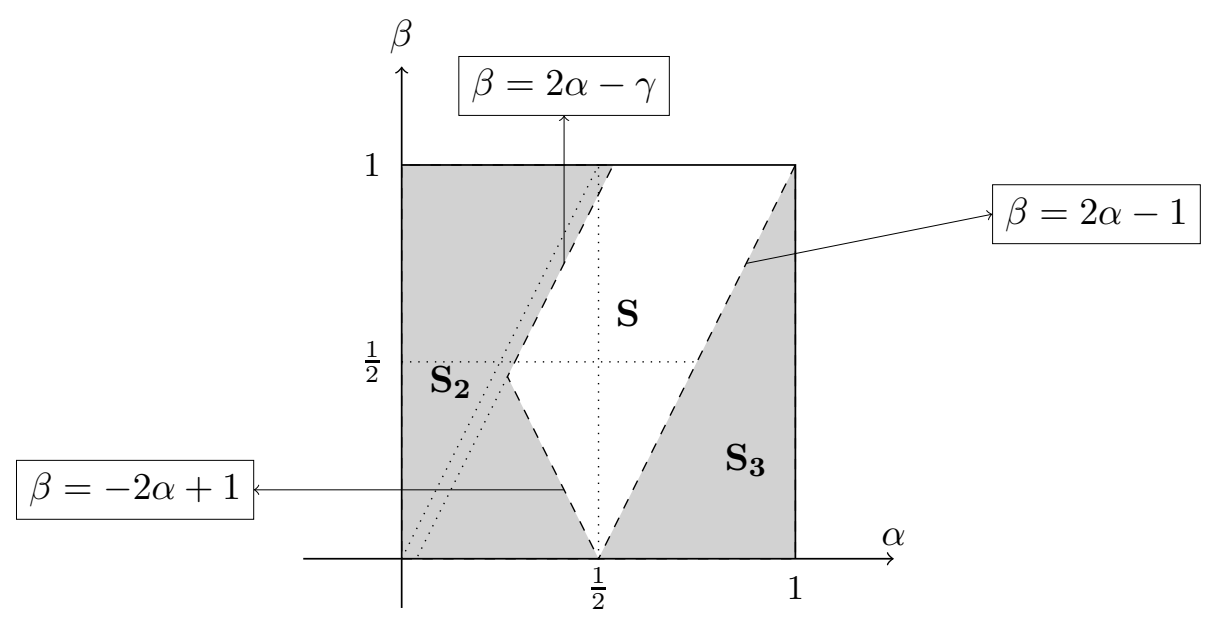

Figure 3.1: Region of non exponential stability for Fourier.

$$
\rho=\mu=\sigma=m=c=\kappa=1
$$

We make the ansatz of separation of variables via the eigenfunctions $\left(\phi_{j}\right)_{j}$ of $A$,

$$
u_{j}(t)=a_{j}(t) \phi_{j}, \theta_{j}(t)=b_{j}(t) \phi_{j}
$$

for arbitrary $j$ to find solutions with decay contradicting exponential stability. Then, solving (1.1), (1.2) is equivalent to solving the following system of ODEs for the coefficient functions 
$\left(a_{j}, b_{j}\right)$

$$
\begin{aligned}
a_{j}^{\prime \prime}+\lambda_{j}^{\gamma} a_{j}^{\prime \prime}+\lambda_{j} a_{j}-\lambda_{j}^{\alpha} b_{j} & =0, \\
b_{j}^{\prime}+\lambda_{j}^{\beta} b_{j}+\lambda_{j}^{\alpha} a_{j}^{\prime} & =0 .
\end{aligned}
$$

System (3.2), (3.3) is equivalent to a first-order system for the column vector $V_{j}:=\left(a_{j}, a_{j}^{\prime}, b_{j}\right)$,

$$
V_{j}^{\prime}=\left(\begin{array}{ccc}
0 & 1 & 0 \\
\frac{-\lambda_{j}}{1+\lambda_{j}^{\gamma}} & 0 & \frac{\lambda_{j}^{\alpha}}{1+\lambda_{j}^{\gamma}} \\
0 & -\lambda_{j}^{\alpha} & -\lambda_{j}^{\beta}
\end{array}\right) V_{j} \equiv A_{j} V_{j} .
$$

We are looking for solutions to (3.4) of type

$$
V_{j}(t)=\mathrm{e}^{\omega_{j} t} V_{j}^{0}
$$

I.e., $\omega_{j}$ has to be an eigenvalue of $A_{j}$ with eigenvector $V_{j}^{0}$ as initial data.

It is the aim to demonstrate that, for any given small $\varepsilon>0$, we have some $j$ and some eigenvalue $\omega_{j}$ such that the real part $\Re \omega_{j}$ of $\omega_{j}$ is larger than $-\varepsilon$. This will contradict the exponential stability (being a kind of uniform property over all initial values). Computing the characteristic polynomial of $A_{j}$ we have

$$
\operatorname{det}\left(\omega-A_{j}\right)=\omega^{3}+\lambda_{j}^{\beta} \omega^{2}+\frac{\lambda_{j}^{2 \alpha}+\lambda_{j}}{1+\lambda_{j}^{\gamma}} \omega+\frac{\lambda_{j}^{1+\beta}}{1+\lambda_{j}^{\gamma}}=: \quad P_{j}(\omega) .
$$

In order to show that

$$
\forall \varepsilon>0 \exists j \exists \omega_{j}, P_{j}\left(\omega_{j}\right)=0: \Re \omega_{j} \geq-\varepsilon,
$$

we introduce, for small $\varepsilon>0$,

$$
z:=\omega+\varepsilon, \quad P_{j, \varepsilon}(z):=P_{j}(z-\varepsilon),
$$

and we have to show

$$
\forall 0<\varepsilon \ll 1 \exists j \exists z_{j}, P_{j, \varepsilon}\left(z_{j}\right)=0: \Re z_{j} \geq 0 .
$$

To prove (3.5) we start with computing

$$
P_{j, \varepsilon(z)}=q_{3} z^{3}+q_{2} z^{2}+q_{1} z+q_{0}
$$

where

$$
\begin{aligned}
& q_{3}=1, \\
& q_{2}=-3 \varepsilon+\lambda_{j}^{\beta}, \\
& q_{1}=3 \varepsilon^{2}-2 \varepsilon \lambda_{j}^{\beta}+\frac{\lambda_{j}^{2 \alpha}+\lambda_{j}}{1+\lambda_{j}^{\gamma}}, \\
& q_{0}=-\varepsilon^{3}+\lambda_{j}^{\beta} \varepsilon^{2}-\frac{\lambda_{j}^{2 \alpha}+\lambda_{j}}{1+\lambda_{j}^{\gamma}} \varepsilon+\frac{\lambda_{j}^{1+\beta}}{1+\lambda_{j}^{\gamma}} .
\end{aligned}
$$


We use the Hurwitz criterion [33]: Let

$$
\mathbb{H}^{j}:=\left(\begin{array}{ccc}
q_{2} & q_{3} & 0 \\
q_{0} & q_{1} & q_{2} \\
0 & 0 & q_{0}
\end{array}\right)
$$

denote the Hurwitz matrix associated to the polynomial $P_{j, \varepsilon}$. Then (3.5) is fulfilled if we find, for given small $\varepsilon>0$, a (sufficiently large) index $j$ such that one of the principal minors of $\mathbb{H}^{j}$ is not positive. The principal minors are given by the determinants $\operatorname{det} D_{m}^{j}$ of the matrices $D_{m}^{j}$, for $m=1,2,3$, where $D_{m}^{j}$ denotes the upper left square submatrix of $\mathbb{H}^{j}$ consisting of the elements $\mathbb{H}_{11}^{j}, \ldots, \mathbb{H}_{m m}^{j}$.

Assuming w.l.o.g. from now on that for some small $\varepsilon_{0}$

$$
0<\varepsilon \leq \varepsilon_{0}<1
$$

Then, for $j$ large enough and for all $\beta \in[0,1]$ we have

$$
\operatorname{det} D_{1}^{j}=q_{2}=-3 \varepsilon+\lambda_{j}^{\beta}>0 .
$$

Consequently, we need to prove

$$
\operatorname{det} D_{2}^{j} \leq 0 \quad \text { or } \quad \operatorname{det} D_{3}^{j} \leq 0
$$

for sufficiently large $j$. Moreover, since $\operatorname{det} D_{3}^{j}=q_{0} \operatorname{det} D_{2}^{j}$, it is sufficient to analyse the signs of $q_{0}$ and $\operatorname{det} D_{2}^{j}$ for sufficiently large $j$.

First, for the sign of $q_{0}$, note that

$$
q_{0}=\frac{1}{1+\lambda_{j}^{\gamma}} \widetilde{q}_{0}
$$

where $\widetilde{q}_{0}$ is given by

$$
\widetilde{q}_{0}=\left(\lambda_{j}^{\beta} \varepsilon^{2}+\lambda_{j}^{\gamma+\beta} \varepsilon^{2}+\lambda_{j}^{1+\beta}\right)-\left(\lambda_{j}^{\gamma} \varepsilon^{3}+\lambda_{j}^{2 \alpha} \varepsilon+\lambda_{j} \varepsilon+\varepsilon^{3}\right) .
$$

Then, in the following we will analyze the sign of $\widetilde{q}_{0}$ distinguishing several cases.

- For the case $\alpha=\beta=0$, we have

$$
\widetilde{q}_{0}=\lambda_{j}^{\gamma}\left(\varepsilon^{2}-\varepsilon^{3}\right)+\lambda_{j}(1-\varepsilon)+\left(\varepsilon^{2}-\varepsilon-\varepsilon^{3}\right)>0
$$

for some (sufficiently large) $j$.

- For the case $\alpha=0, \beta>0$, we have

$$
\widetilde{q}_{0}=\left(\lambda_{j}^{\beta} \varepsilon^{2}+\lambda_{j}^{\gamma+\beta} \varepsilon^{2}+\lambda_{j}^{1+\beta}\right)-\left(\lambda_{j}^{\gamma} \varepsilon^{3}+\lambda_{j} \varepsilon+\varepsilon+\varepsilon^{3}\right)>0
$$

for some (sufficiently large) $j$, because $\gamma+\beta>\gamma$. 
- For the case $\alpha>0, \beta=0$, we have

$$
\widetilde{q}_{0}=\lambda_{j}^{\gamma}\left(\varepsilon^{2}-\varepsilon^{3}\right)+\lambda_{j}(1-\varepsilon)-\lambda_{j}^{2 \alpha} \varepsilon+\left(\varepsilon^{2}-\varepsilon^{3}\right) .
$$

Then, in this case, the sign of $\widetilde{q}_{0}$ depends on $\alpha$, as we get:

- For $\alpha \in\left(0, \frac{1}{2}\right]$, we have $\widetilde{q}_{0}>0$ for sufficiently large $j$.

- For $\alpha \in\left(\frac{1}{2}, 1\right]$, we have $\widetilde{q}_{0}<0$ for sufficiently large $j$.

- For the case $\alpha>0, \beta>0$, we obtain:

1. For $\alpha \in\left(0, \frac{1}{2}\right]$, we have $2 \alpha \leq 1<1+\beta$ and $\gamma \leq 1<1+\beta$. Then $\widetilde{q}_{0}>0$ for sufficiently large $j$; the dominant term is $\lambda_{j}^{1+\beta}$.

2. For $\alpha \in\left(\frac{1}{2}, 1\right]$ the sign of $\widetilde{q}_{0}$ depends on the relationship between $1+\beta$ and $2 \alpha$. In fact:

(a) For $1+\beta \geq 2 \alpha$ we have $\widetilde{q}_{0}>0$ for sufficiently large $j$; the dominant term is $\lambda_{j}^{1+\beta}$.

(b) For $1+\beta<2 \alpha$ we have $\widetilde{q}_{0}<0$ for sufficiently large $j$; the dominant term is $-\lambda_{j}^{2 \alpha} \varepsilon$.

Therefore, combining all cases we have the regions described in figure 3.2, the regions being independent of $\gamma$.

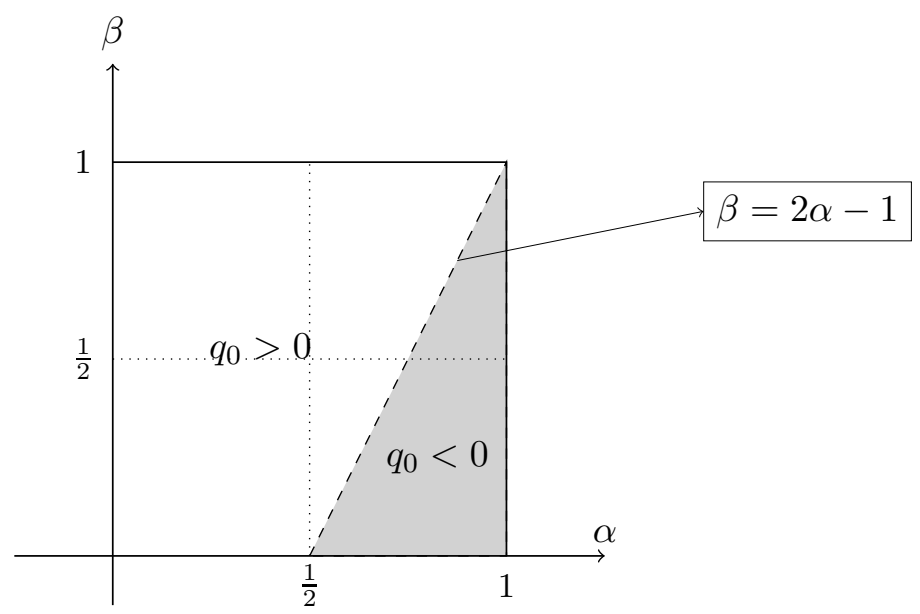

Figure 3.2: Region of $q_{0}<0$.

Second, for the sign of $\operatorname{det} D_{2}^{j}$, note that

$$
\operatorname{det} D_{2}^{j}=\frac{1}{1+\lambda_{j}^{\gamma}} d_{2}^{j},
$$

where $d_{2}^{j}$ is given by

$$
\begin{aligned}
d_{2}^{j}= & \left(\left(6 \varepsilon^{3}+2 \varepsilon^{2}\right) \lambda_{j}^{\beta}+\left(6 \varepsilon^{3}+2 \varepsilon^{2}\right) \lambda_{j}^{\gamma+\beta}+\lambda_{j}^{2 \alpha+\beta}\right) \\
& -\left(2 \varepsilon \lambda_{j}^{2 \beta}+8 \varepsilon^{3} \lambda_{j}^{\gamma}+2 \varepsilon \lambda_{j}^{2 \beta+\gamma}+2 \varepsilon \lambda_{j}^{2 \alpha}+2 \varepsilon \lambda_{j}+8 \varepsilon^{3}\right) .
\end{aligned}
$$


Hence, we will analyze the sign of $d_{2}^{j}$ in the same cases as to $q_{0}$.

- For the case $\alpha=\beta=0$, we have

$$
d_{2}^{j}=-\varepsilon\left(2-2 \varepsilon+2 \varepsilon^{2}\right) \lambda_{j}^{\gamma}-2 \varepsilon \lambda_{j}+\left(1-\left(4 \varepsilon+2 \varepsilon^{3}-2 \varepsilon^{2}\right)\right)<0
$$

for sufficiently large $j$, independent of $\gamma$.

- For the case $\alpha=0, \beta>0$, we have

$$
\begin{aligned}
d_{2}^{j}= & \left(\left(1+6 \varepsilon^{3}+2 \varepsilon^{2}\right) \lambda_{j}^{\beta}+\left(6 \varepsilon^{3}+2 \varepsilon^{2}\right) \lambda_{j}^{\gamma+\beta}\right) \\
& -\left(2 \varepsilon \lambda_{j}^{2 \beta}+8 \varepsilon^{3} \lambda_{j}^{\gamma}+2 \varepsilon \lambda_{j}^{2 \beta+\gamma}+2 \varepsilon \lambda_{j}+\left(2 \varepsilon+8 \varepsilon^{3}\right)\right)<0
\end{aligned}
$$

for sufficiently large $j$, because $2 \beta+\gamma>\gamma+\beta>\beta$.

- For the case $\alpha>0, \beta=0$, we have

$$
d_{2}^{j}=(1-2 \varepsilon) \lambda_{j}^{2 \alpha}-2 \varepsilon \lambda_{j}-\left(2 \varepsilon-2 \varepsilon^{2}+2 \varepsilon^{3}\right) \lambda_{j}^{\gamma}-\left(2 \varepsilon-2 \varepsilon^{2}+2 \varepsilon^{3}\right) .
$$

1. For $\alpha \in\left(0, \frac{1}{2}\right)$, we have $d_{2}^{j}<0$ for sufficiently large $j$.

2. For $\alpha \in\left[\frac{1}{2}, 1\right]$, we have $d_{2}^{j}>0$ for sufficiently large $j$.

- For the case $\alpha>0, \beta>0$, the sign of $d_{2}^{j}$ depends of the relationship between $2 \alpha$ and $\gamma$. In fact:

1. For $2 \alpha<\gamma$ and for some (sufficiently large) $j$, we have $d_{2}^{j}<0$ because

$$
\beta<2 \alpha+\beta<\gamma+\beta<2 \beta+\gamma,
$$

i.e. the domain term is $-2 \varepsilon \lambda_{j}^{2 \beta+\gamma}$.

2. For $2 \alpha \geq \gamma$ we have two situations:

(a) If $2 \alpha+\beta \geq \max \{2 \beta+\gamma, 2 \alpha, 1\}$ we have $d_{2}^{j}>0$ for sufficiently large $j$. The dominant term is $\lambda_{j}^{2 \alpha+\beta}$. We have

$$
2 \alpha-\beta \geq \gamma \quad \text { and } \quad 2 \alpha+\beta \geq 1 .
$$

(b) If $2 \alpha+\beta<\max \{2 \beta+\gamma, 2 \alpha, 1\}$ we have $d_{2}^{j}<0$ for sufficiently large $j$. The dominant term is $-2 \varepsilon\left(\lambda_{j}^{2 \beta+\gamma}+\lambda_{j}^{2 \alpha}+\lambda_{j}\right)$. We have

$$
2 \alpha-\beta<\gamma \quad \text { or } \quad 2 \alpha+\beta<1 \text {. }
$$

Therefore, combining all cases in Subsection 3.2 we have the figure 3.3.

Finally, summarizing all cases, see graphics 3.2 and 3.3, we can deduce that the negative values of det $D_{2}^{j}$ and $\operatorname{det} D_{3}^{j}$ (for sufficiently large $j$ ), are given in the following figure 3.4. Therefore, we have proved the following theorem.

Theorem 3.1. The region of non-exponential stability for the Fourier model (1.1), (1.2) is given by the complement of the set $S$, see (2.13), which is precisely shown in Figure 3.4. 


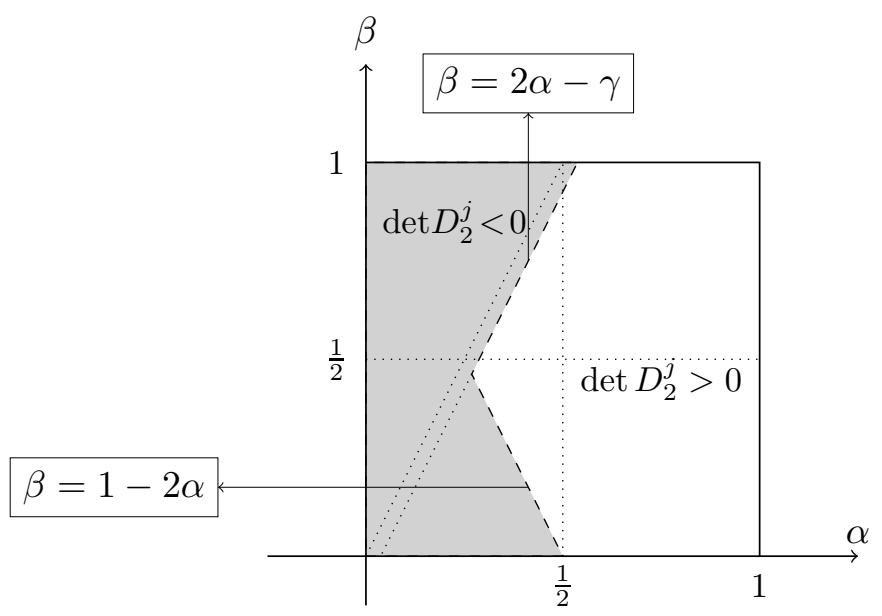

Figure 3.3: Region of $\operatorname{det} D_{2}^{j}>0$.

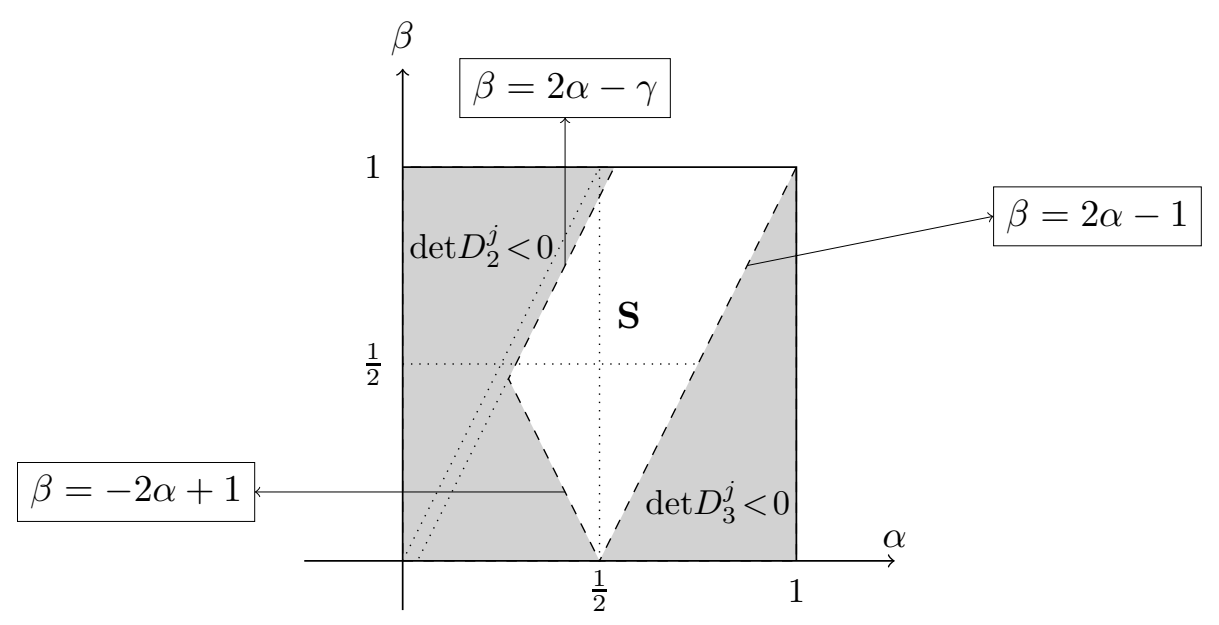

Figure 3.4: Region of non-exponential stability.

\section{Loss of exponential stability for the Cattaneo law}

The known examples on the loss of exponential stability described above, like the Timoshenko system or the classical thermoelastic plate corresponding to $(\alpha, \beta, \gamma)=\frac{1}{2}(1,1,1)$ in $(1.4)-(1.6)$ might have been regarded as exceptions, in view of the pure heat conduction system, or in view of the classical second-order thermeolastic system, where both for the Fourier and for the Cattaneo model exponential stability is given. In this section we demonstrate that for a large class of systems exponential stability is lost when replacing Fourier's law by Cattaneo's law, first without $(\gamma=0)$ then with inertial term $(\gamma>0)$.

We start considering both cases simultaneously, i.e. $\mu, \gamma \geq 0$. Without loss of generality we assume

$$
\rho_{1}=\rho_{2}=m=\kappa=1 \text {. }
$$


As in Section 3 we make the ansatz of separation of variables via the eigenfunctions $\left(\phi_{j}\right)_{j}$ of $A$,

$$
u_{j}(t)=a_{j}(t) \phi_{j}, \theta_{j}(t)=b_{j}(t) \phi_{j}, q_{j}(t)=c_{j}(t) B_{2} \phi_{j}
$$

for arbitrary $j$ (assuming $B_{2} \phi_{j}$ to be not identically zero), to find solutions with decay contradicting exponential stability.

Using (1.7) we observe

$$
B_{1} q_{j}(t)=c_{j}(t) B_{1} B_{2} \phi_{j}=-c_{j}(t) A^{\beta} \phi_{j}=-\lambda_{j}^{\beta} c_{j}(t) \phi_{j}
$$

thus solving (1.4)-(1.6) is equivalent to solving the following system of ODEs for the coefficient functions $\left(a_{j}, b_{j}, c_{j}\right)$, where a prime' denotes again differentiation with respect to time $t$,

$$
\begin{aligned}
a_{j}^{\prime \prime}+\mu \lambda_{j}^{\gamma} a_{j}^{\prime \prime}+\lambda_{j} a_{j}-\lambda_{j}^{\alpha} b_{j} & =0, \\
b_{j}^{\prime}-\lambda_{j}^{\beta} c_{j}+\lambda_{j}^{\alpha} a_{j}^{\prime} & =0, \\
\tau c_{j}^{\prime}+c_{j}+b_{j} & =0 .
\end{aligned}
$$

The last equation arises from (1.6) with the ansatz (4.1) using again the natural condition that $B_{2} \phi_{j}$ is not identically zero.

System (4.2)-(4.4) is equivalent to a first-order system for the column vector $V_{j}:=\left(a_{j}, a_{j}^{\prime}, b_{j}, c_{j}\right)$,

$$
V_{j}^{\prime}=\left(\begin{array}{cccc}
0 & 1 & 0 & 0 \\
\frac{-\lambda_{j}}{1+\mu \lambda_{j}^{\gamma}} & 0 & \frac{\lambda_{j}^{\alpha}}{1+\mu \lambda_{j}^{\gamma}} & 0 \\
0 & -\lambda_{j}^{\alpha} & 0 & \lambda_{j}^{\beta} \\
0 & 0 & -\frac{1}{\tau} & -\frac{1}{\tau}
\end{array}\right) V_{j} \equiv A_{j} V_{j}
$$

We are looking for solutions to (4.5) of type

$$
V_{j}(t)=\mathrm{e}^{\omega_{j} t} V_{j}^{0}
$$

i.e., $\omega_{j}$ has to be an eigenvalue of $A_{j}$ with eigenvector $V_{j}^{0}$ as initial data.

It is the aim to demonstrate that, for any given small $\varepsilon>0$, we have some $j$ and some eigenvalue $\omega_{j}$ such that the real part $\Re \omega_{j}$ of $\omega_{j}$ is larger than $-\varepsilon$. This will contradict the exponential stability (being a kind of uniform property over all initial values). Computing the characteristic polynomial of $A_{j}$ we have

$$
\begin{aligned}
\operatorname{det}\left(A_{j}-\omega\right)= & \frac{1}{\tau\left(1+\mu \lambda_{j}^{\gamma}\right)}\left(\left[\tau\left(1+\mu \lambda_{j}^{\gamma}\right)\right] \omega^{4}+\left[1+\mu \lambda_{j}^{\gamma}\right] \omega^{3}+\right. \\
& {\left.\left[\lambda_{j}^{\beta}\left(1+\mu \lambda_{j}^{\gamma}\right)+\tau\left(\lambda_{j}+\lambda_{j}^{2 \alpha}\right)\right] \omega^{2}+\left[\lambda_{j}+\lambda_{j}^{2 \alpha}\right] \omega+\lambda_{j}^{1+\beta}\right) } \\
\equiv & \frac{1}{\tau\left(1+\mu \lambda_{j}^{\gamma}\right)} P_{j}(\omega) .
\end{aligned}
$$

In order to show that

$$
\forall \varepsilon>0 \exists j \exists \omega_{j}, P_{j}\left(\omega_{j}\right)=0: \Re \omega_{j} \geq-\varepsilon,
$$


we introduce, for small $\varepsilon>0$,

$$
z:=\omega+\varepsilon, \quad P_{j, \varepsilon}(z):=P_{j}(z-\varepsilon),
$$

and we have to show

$$
\forall 0<\varepsilon \ll 1 \exists j \exists z_{j}, P_{j, \varepsilon}\left(z_{j}\right)=0: \Re z_{j} \geq 0 .
$$

To prove (4.6) we start with computing

$$
P_{j, \varepsilon}(z)=q_{4} z^{4}+q_{3} z^{3}+q_{2} z^{2}+q_{1} z+q_{0}
$$

where

$$
\begin{aligned}
& q_{4}=\tau\left(1+\mu \lambda_{j}^{\gamma}\right), \\
& q_{3}=(1-4 \tau \varepsilon)\left(1+\mu \lambda_{j}^{\gamma}\right), \\
& q_{2}=6 \tau \varepsilon^{2}\left(1+\mu \lambda_{j}^{\gamma}\right)-3 \varepsilon\left(1+\mu \lambda_{j}^{\gamma}\right)+\lambda_{j}^{\beta}\left(1+\mu \lambda_{j}^{\gamma}\right)+\tau\left(\lambda_{j}+\lambda_{j}^{2 \alpha}\right), \\
& q_{1}=-4 \tau \varepsilon^{3}\left(1+\mu \lambda_{j}^{\gamma}\right)+3 \varepsilon^{2}\left(1+\mu \lambda_{j}^{\gamma}\right)-2\left(\lambda_{j}^{\beta}\left(1+\mu \lambda_{j}^{\gamma}\right)+\tau\left(\lambda_{j}+\lambda_{j}^{2 \alpha}\right)\right) \varepsilon+\lambda_{j}+\lambda_{j}^{2 \alpha}, \\
& q_{0}=\tau \varepsilon^{4}\left(1+\mu \lambda_{j}^{\gamma}\right)-\varepsilon^{3}\left(1+\mu \lambda_{j}^{\gamma}\right)+\left(\lambda_{j}^{\beta}\left(1+\mu \lambda_{j}^{\gamma}\right)+\tau\left(\lambda_{j}+\lambda_{j}^{2 \alpha}\right)\right) \varepsilon^{2}-\left(\lambda_{j}+\lambda_{j}^{2 \alpha}\right) \varepsilon+\lambda_{j}^{1+\beta} .
\end{aligned}
$$

As in Section 3 we use the Hurwitz criterion. Let

$$
\mathbb{H}^{j}:=\left(\begin{array}{cccc}
q_{3} & q_{4} & 0 & 0 \\
q_{1} & q_{2} & q_{3} & q_{4} \\
0 & q_{0} & q_{1} & q_{2} \\
0 & 0 & 0 & q_{0}
\end{array}\right)
$$

denote the Hurwitz matrix associated to the polynomial $P_{j, \varepsilon}$. Then (4.6) is fulfilled if we find, for given small $\varepsilon>0$, a (sufficiently large) index $j$ such that one of the principal minors of $\mathbb{H}^{j}$ is not positive. The principal minors are given by the determinants $\operatorname{det} D_{m}^{j}$ of the matrices $D_{m}^{j}$, for $m=1,2,3,4$, where $D_{m}^{j}$ denotes the upper left square submatrix of $\mathbb{H}^{j}$ consisting of the elements $\mathbb{H}_{11}^{j}, \ldots, \mathbb{H}_{m m}^{j}$. We assume w.l.o.g. from now on that

$$
0<\varepsilon \leq \varepsilon_{0}<\frac{1}{4 \tau}
$$

Hence we have in the considered region $q_{4}, q_{3}, q_{2}>0$ for $j$ large enough, implying

$$
\operatorname{det} D_{1}^{j}=q_{3}>0 \text {. }
$$

Now we have to distinguish the two cases $\gamma=0$ and $\gamma>0$.

\subsection{Case $^{1} \gamma=0$}

For the case $\gamma=0$ we may also assume w.l.o.g. $\mu=0$. Exponential stability is known for the Fourier case $(\tau=0)$ in the striped region

$$
\mathcal{S}_{e s}(\tau=0, \gamma=0):=\{(\beta, \alpha) \mid 1-2 \beta \leq \alpha \leq 2 \beta, \alpha \geq 2 \beta-1\},
$$




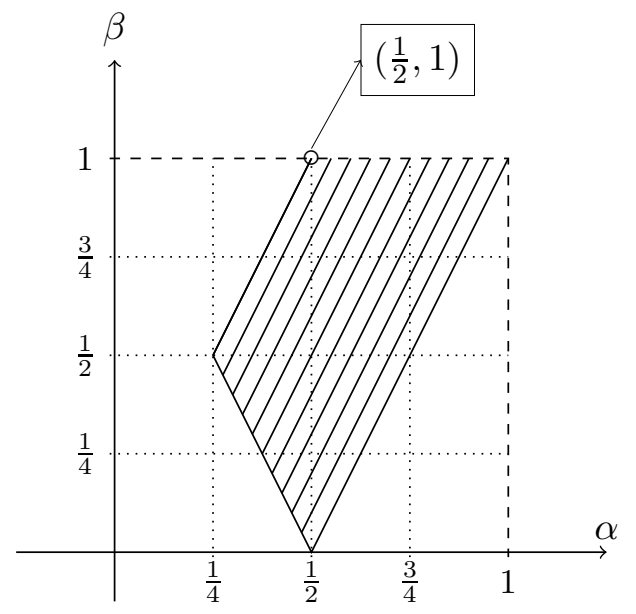

Figure 4.1: $\mathcal{S}_{e s}(\tau=0, \gamma=0)$.

see Figure 4.1. The pair $(\alpha, \beta)=\left(\frac{1}{2}, 1\right)$ is highlighted by a circle since this will be the only pair for which the exponential stability will remain true if we replace the Fourier law by the Cattaneo law. In the remaining part of the striped region of exponential stability for $\tau=0$, the property of exponential stability will be lost. We have

Theorem 4.1. The region of exponential stability given for the Fourier model $(\tau=0)$ by $\mathcal{S}_{e s}(\tau=0, \gamma=0)$ in (4.7) resp. Figure 4.1, is lost for the Cattaneo model ( $\left.\tau>0\right)$ in (1.4)-(1.6) in any point different from the singular point $(\alpha, \beta)=\left(\frac{1}{2}, 1\right)$, see Figure 4.2.

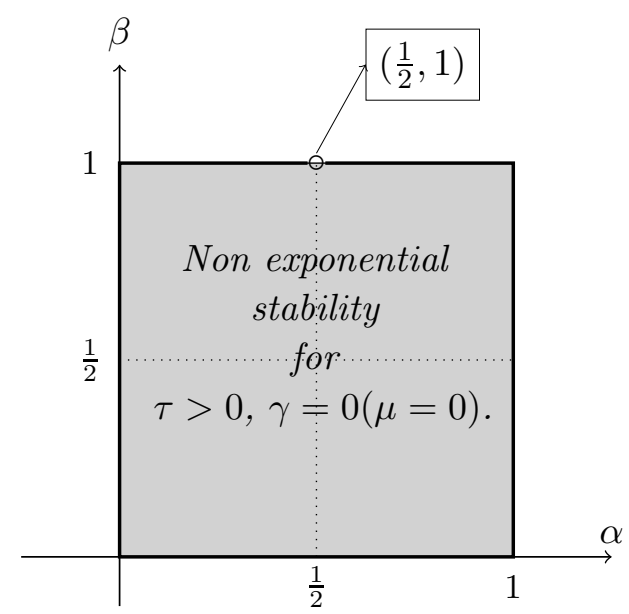

Figure 4.2: Region of non exponential stability for $\tau>0, \gamma=0(\mu=0)$.

Proof. The exponential stability of system for $(\alpha, \beta)=\left(\frac{1}{2}, 1\right)$ (and $\tau>0$ from now on) has

\footnotetext{
${ }^{1}$ The results in this subsection have been partially announced in [30]
} 
been proved for the realization given in Example 3 from the introduction for various boundary conditions [28].

Then, in order to obtain our result, we will prove that

$$
\operatorname{det} D_{2}^{j} \leq 0 \quad \text { or } \operatorname{det} D_{3}^{j} \leq 0
$$

in $[0,1] \times[0,1] \backslash\left(\frac{1}{2}, 1\right)$, for some (sufficiently large) $j$. The set of parameters $(\alpha, \beta)$ for which we have to prove this, will be divided into three subsets, the first one, where $\beta<1$, the second, where $\beta=1$ and $\frac{1}{2}<\alpha \leq 1$, and the third one, where $\beta=1$ and $0 \leq \alpha<\frac{1}{2}$ :

Part I: $\beta<1$.

We have

$$
\begin{aligned}
\operatorname{det} D_{2}^{j}= & q_{3} q_{2}-q_{4} q_{1} \\
= & {[1-4 \tau \varepsilon] \cdot\left[6 \tau \varepsilon^{2}-3 \varepsilon+\lambda_{j}^{\beta}+\tau\left(\lambda_{j}+\lambda_{j}^{2 \alpha}\right)\right]-} \\
& \tau\left[-4 \tau \varepsilon^{3}+3 \varepsilon^{2}-2\left(\lambda_{j}^{\beta}+\tau\left(\lambda_{j}+\lambda_{j}^{2 \alpha}\right)\right) \varepsilon+\lambda_{j}+\lambda_{j}^{2 \alpha}\right] \\
= & -2 \tau^{2} \varepsilon \lambda_{j}^{2 \alpha}-2 \tau^{2} \varepsilon \lambda_{j}+(1-2 \tau \varepsilon) \lambda_{j}^{\beta}-20 \tau^{2} \varepsilon^{3}+15 \tau \varepsilon^{2}-3 \varepsilon
\end{aligned}
$$

implying

$$
\operatorname{det} D_{2}^{j} \leq-2 \tau^{2} \varepsilon \lambda_{j}+\mathcal{O}\left(\lambda_{j}^{\beta}\right),
$$

where we use the Landau symbol $\mathcal{O}\left(\lambda_{j}^{\beta}\right)$ to denote a term satisfying

$$
\left.\mid \mathcal{O}\left(\lambda_{j}^{\beta}\right)\right) \mid \leq k_{1} \lambda_{j}^{\beta}
$$

with a positive constant $k_{1}$ (being independent of $j, \varepsilon, \tau$ ). Thus we conclude from (4.9)

$$
\operatorname{det} D_{2}^{j}<0
$$

for sufficiently large $j$ (depending on $\varepsilon, \tau$ ) since $\beta<1$ and $\lambda_{j} \rightarrow \infty$ by assumption.

Part II: $\beta=1, \frac{1}{2}<\alpha \leq 1$.

In this case, from (4.8) we have

$$
\operatorname{det} D_{2}^{j}=-2 \tau^{2} \varepsilon \lambda_{j}^{2 \alpha}+\left(1-2 \tau \varepsilon-2 \tau^{2}\right) \lambda_{j}-20 \tau^{2} \varepsilon^{3}+15 \tau \varepsilon^{2}-3 \varepsilon
$$

implying

$$
\operatorname{det} D_{2}^{j} \leq-2 \tau^{2} \varepsilon \lambda_{j}^{2 \alpha}+\mathcal{O}\left(\lambda_{j}\right)
$$

Thus we conclude that

$$
\operatorname{det} D_{2}^{j}<0
$$

for sufficiently large $j$ (depending on $\varepsilon, \tau$ ) since $2 \alpha>1$ and $\lambda_{j} \rightarrow \infty$.

Part III: $\beta=1,0 \leq \alpha<\frac{1}{2}$. 
Here, note that

$$
\operatorname{det} D_{3}^{j}=q_{1} \operatorname{det} D_{2}^{j}-q_{3}^{2} q_{0}
$$

Then, computing each term in (4.12) with $\beta=1$, we have

$$
\begin{aligned}
\operatorname{det} D_{2}^{j} & =-2 \tau^{2} \varepsilon \lambda_{j}^{2 \alpha}+[1-2 \tau \varepsilon(1+\tau)] \lambda_{j}-20 \tau^{2} \varepsilon^{3}+15 \tau \varepsilon^{2}-3 \varepsilon \\
q_{1} & =(1-2 \tau \varepsilon) \lambda_{j}^{2 \alpha}+[1-2 \varepsilon(1+\tau)] \lambda_{j}+3 \varepsilon^{2}-4 \tau \varepsilon^{3} \\
q_{0} & =-\varepsilon(1-\tau \varepsilon) \lambda_{j}^{2 \alpha}-\varepsilon[1-\varepsilon(1+\tau)] \lambda_{j}+\lambda_{j}^{2}-\varepsilon^{3}+\tau \varepsilon^{4} \\
q_{3} & =(1-4 \tau \varepsilon),
\end{aligned}
$$

which implies, using $0 \leq 2 \alpha<1$ :

$$
\begin{aligned}
\operatorname{det} D_{2}^{j} & =[1-2 \tau \varepsilon(1+\tau)] \lambda_{j}+\mathcal{O}\left(\lambda_{j}^{2 \alpha}\right) \\
q_{1} & =[1-2 \varepsilon(1+\tau)] \lambda_{j}+\mathcal{O}\left(\lambda_{j}^{2 \alpha}\right) \\
q_{0} & =\lambda_{j}^{2}+\mathcal{O}\left(\lambda_{j}\right) \\
q_{3} & =(1-4 \tau \varepsilon) .
\end{aligned}
$$

Then, in (4.12) we have

$$
\operatorname{det} D_{3}^{j}=\left[(1-2 \tau \varepsilon(1+\tau))(1-2 \varepsilon(1+\tau))-(1-4 \tau \varepsilon)^{2}\right] \lambda_{j}^{2}+\mathcal{O}\left(\lambda_{j}^{2 \alpha+1}\right),
$$

which implies

$$
\operatorname{det} D_{3}^{j}=-2 \varepsilon(1-2 \tau \varepsilon)(1-\tau)^{2} \lambda_{j}^{2}+\mathcal{O}\left(\lambda_{j}^{2 \alpha+1}\right) .
$$

Thus, we conclude that

$$
\operatorname{det} D_{3}^{j}<0
$$

for sufficiently large $j$ (depending on $\varepsilon, \tau$ ) since $2 \alpha<1$ and $\lambda_{j} \rightarrow \infty$.

With (4.10), (4.11) and (4.13) we have proved (4.6) and hence Theorem 4.1.

\subsection{Case $\gamma>0$}

Now we consider the system (1.4)-(1.6) with inertial term, i.e. with $\mu, \gamma>0$. With another more sophisticated analysis using again the Hurwitz criterion we can prove

Theorem 4.2. The region of exponential stability given for the Fourier model (1.1), (1.2) by $S$ in (2.13), is lost for the Cattaneo model (1.4)-(1.6) in any point not belonging to the exceptional set

$$
\mathcal{S}_{\text {exc }}:=\left\{(\alpha, \beta, \gamma) \in[0,1]^{3} \mid \alpha \geq 1 / 2, \gamma+\beta=2 \alpha\right\} .
$$

Remark 4.3. In the exceptional set exponential stability is kept in general, see Theorem 4.5.

Remark 4.4. For $\gamma=0$ the exceptional set $\mathcal{S}_{\text {exc }}$ reduces to the exceptional singular point $\{(\alpha, \beta)=(1 / 2,1)\}$, as known from the previous subsection. 
Proof of Theorem 4.2:

We first check the positivity of the coefficients $q_{1}$ and $q_{0}$. Since

$$
q_{1}=\mu \lambda_{j}^{\gamma}\left(3 \varepsilon^{2}-4 \varepsilon^{3} \tau\right)+\lambda_{j}^{2 \alpha}(1-2 \varepsilon \tau)+\lambda_{j}(1-2 \varepsilon \tau)-2 \mu \varepsilon \lambda_{j}^{\beta+\gamma}-2 \varepsilon \lambda_{j}^{\beta}
$$

and

$$
\beta<\beta+\gamma \leq 2 \alpha
$$

we have

$$
q_{1}>0
$$

if $\varepsilon$ is small enough and $j$ is large enough. Similarly, for

$$
q_{0}=\lambda_{j}^{1+\beta}+\varepsilon^{2} \mu \lambda_{j}^{\gamma+\beta}+\varepsilon^{2} \lambda_{j}^{\beta}-\mu \lambda_{j}^{\gamma}\left(\varepsilon^{3}-\varepsilon^{4} \tau\right)-\lambda_{j}^{2 \alpha}\left(\varepsilon-\varepsilon^{2} \tau\right)-\lambda_{j}\left(\varepsilon-\varepsilon^{2} \tau\right),
$$

since

$$
\gamma \leq \max \{1,2 \alpha, \gamma+\beta\} \leq 1+\beta
$$

we have

$$
q_{0}>0
$$

The relation

$$
\operatorname{det} D_{4}^{j}=q_{0} \operatorname{det} D_{3}^{j}
$$

requires us to show that

$$
\operatorname{det} D_{2}^{j} \leq 0 \quad \text { or } \quad \operatorname{det} D_{3}^{j} \leq 0
$$

We compute

$$
\begin{aligned}
\operatorname{det} D_{2}^{j}= & q_{3} q_{2}-q_{1} q_{4} \\
= & -\lambda_{j}^{2 \gamma}\left[\mu^{2}(1-4 \tau \varepsilon)\left(3 \varepsilon-6 \varepsilon^{2}\right)+\mu^{2} \tau\left(3 \varepsilon^{2}-4 \varepsilon^{3} \tau\right)\right]-\lambda_{j}^{\gamma+2 \alpha}[2 \mu \tau \varepsilon] \\
& -\lambda_{j}^{\gamma+1}[2 \mu \tau \varepsilon]-\lambda_{j}^{\gamma}\left[\tau \mu\left(3 \varepsilon^{2}-4 \varepsilon^{3} \tau\right)+2 \mu(1-4 \tau \varepsilon)\left(3 \varepsilon-6 \varepsilon^{2} \tau\right)\right] \\
& -\lambda_{j}^{2 \alpha}[2 \tau \varepsilon]-\lambda_{j}[2 \tau \varepsilon]-\left[(1-4 \tau \varepsilon)\left(3 \varepsilon-6 \varepsilon^{2} \tau\right)\right] \\
& +\lambda_{j}^{2 \gamma+\beta}\left[\mu^{2}(1-2 \tau \varepsilon)\right]+\lambda_{j}^{\gamma+\beta}[2 \mu(1-2 \tau \varepsilon)]+\lambda_{j}^{\beta}[1-2 \tau \varepsilon] .
\end{aligned}
$$

In order to compare the negative and the positive terms, we distinguish the following cases:

$$
\text { Case I: } \quad 2 \gamma+\beta \leq 1 .
$$

Then, since

$$
\beta<\gamma+\beta<2 \gamma+\beta \leq 1<1+\gamma
$$

we have

$$
\begin{aligned}
\operatorname{det} D_{2}^{j} & \leq-2 \mu \tau \varepsilon \lambda_{j}^{\gamma+1}+\mathcal{O}\left(\lambda_{j}\right) \\
& <0
\end{aligned}
$$


as $j \rightarrow \infty$, implying non-exponential stability.

$$
\text { Case II: } \quad 2 \gamma+\beta>1, \quad \gamma+\beta<2 \alpha .
$$

Then

$$
\begin{aligned}
\operatorname{det} D_{2}^{j} & \leq-2 \mu \tau \varepsilon \lambda_{j}^{\gamma+2 \alpha}+\mathcal{O}\left(\lambda_{j}^{2 \gamma+\beta}\right) \\
& <0
\end{aligned}
$$

as $j \rightarrow \infty$.

$$
\text { Case III: } \quad 2 \gamma+\beta>1, \quad \alpha<\frac{1}{2}
$$

Then

$$
\begin{aligned}
\operatorname{det} D_{2}^{j} & \leq-2 \mu \tau \varepsilon \lambda_{j}^{\gamma+1}+\mathcal{O}\left(\lambda_{j}^{2 \gamma+\beta}\right) \\
& <0
\end{aligned}
$$

as $j \rightarrow \infty$. Finally,

$$
\text { Case IV: } \quad 2 \gamma+\beta>1, \quad \gamma+\beta=2 \alpha, \quad \alpha \geq \frac{1}{2} .
$$

This describes the exceptional set. We remark that in this case one can compute

$$
\operatorname{det} D_{2}^{j}>0, \quad \operatorname{det} D_{3}^{j}>0
$$

indicating exponential stability.

Thus we have proved Theorem 4.2.

\subsection{Exponential stability in $\mathrm{S}_{\mathrm{exc}}$}

Here we study the exponential stability of system (1.4)-(1.6) in the exceptional set $S_{\text {exc }}$ defined in (4.14). Together with condition (1.7) we additionally assume the coupling condition

$$
B_{1}^{*}=-\frac{1}{\kappa} B_{2} \quad \text { with } D\left(B_{2}\right)=D\left(A^{\beta / 2}\right),
$$

implying

$$
\left\|B_{2} \phi\right\|=\left\|A^{\beta / 2} \phi\right\| .
$$

We obtain for a regular solution, in multiplying (1.1) by $u_{t}$, and (1.2) by $\theta$, the equality

$$
\frac{1}{2} \frac{d}{d t}\left[\rho_{1}\left\|u_{t}\right\|^{2}+\mu\left\|A^{\gamma / 2} u_{t}\right\|^{2}+\sigma\left\|A^{1 / 2} u\right\|^{2}+\rho_{2}\|\theta\|^{2}+\frac{\tau}{\kappa}\|q\|^{2}\right]+\frac{1}{\kappa}\|q\|^{2}=0
$$

This motivates the choice of the Hilbert space

$$
\mathcal{H}=D\left(A^{1 / 2}\right) \times D\left(A^{\gamma / 2}\right) \times H \times[H]^{r}
$$


with inner product defined for $U_{j}=\left(u_{j}, v_{j}, \theta_{j}, q_{j}\right)^{T} \in \mathcal{H}, j=1,2$, as

$$
\left\langle U_{1}, U_{2}\right\rangle_{\mathcal{H}}=\sigma\left(A^{1 / 2} u_{1}, A^{1 / 2} u_{2}\right)+\mu\left(A^{\gamma / 2} v_{1}, A^{\gamma / 2} v_{2}\right)+\rho_{1}\left(v_{1}, v_{2}\right)+\rho_{2}\left(\theta_{1}, \theta_{2}\right)+\frac{\tau}{\kappa}\left(q_{1}, q_{2}\right)_{H^{r}} .
$$

As in Section 2, system (1.4)-(1.6) can be written as an evolution equation in $\mathcal{H}$ given by

$$
\left\{\begin{array}{l}
\frac{d U}{d t}=\mathcal{A}_{\alpha, \beta, \gamma, \tau} U, \\
U(0)=U_{0},
\end{array}\right.
$$

where $U_{0}=\left(u_{0}, v_{0}, \theta_{0}, q_{0}\right)^{T}$, and the operator $\mathcal{A}_{\alpha, \beta, \gamma, \tau}: D\left(\mathcal{A}_{\alpha, \beta, \gamma, \tau}\right) \subset \mathcal{H} \rightarrow \mathcal{H}$ is defined for $\alpha \leq 1 / 2$ by

$$
\mathcal{A}_{\alpha, \beta, \gamma, \tau}\left(\begin{array}{c}
u \\
v \\
\theta \\
q
\end{array}\right)=\left(\begin{array}{c}
v \\
-\left(I+\frac{\mu}{\rho_{1}} A^{\gamma}\right)^{-1} A^{\alpha}\left\{\frac{\sigma}{\rho_{1}} A^{1-\alpha} u-\frac{m}{\rho_{1}} \theta\right\} \\
-\frac{m}{\rho_{2}} A^{\alpha} v-\frac{1}{\rho_{2}} B_{1} q \\
-\frac{1}{\tau} q-\frac{1}{\tau} B_{2} \theta
\end{array}\right)
$$

with domain

$$
\begin{aligned}
D\left(\mathcal{A}_{\alpha, \beta, \gamma, \tau}\right)= & \left\{(u, v, \theta, q)^{T} \in \mathcal{H} \mid v \in D\left(A^{1 / 2}\right), u \in D\left(A^{1-\alpha}\right), \sigma A^{1-\alpha} u-m \theta \in D\left(A^{\alpha-\gamma / 2}\right)\right. \\
& \left.\theta \in D\left(B_{2}\right), B_{1} q \in H\right\} .
\end{aligned}
$$

For $\alpha>1 / 2$ it is defined by

$$
\mathcal{A}_{\alpha, \beta, \gamma, \tau}\left(\begin{array}{c}
u \\
v \\
\theta \\
q
\end{array}\right)=\left(\begin{array}{c}
v \\
-\left(I+\frac{\mu}{\rho_{1}} A^{\gamma}\right)^{-1} A^{\alpha}\left\{\frac{\sigma}{\rho_{1}} A^{1-\alpha} u-\frac{m}{\rho_{1}} \theta\right\} \\
-A^{\alpha-1 / 2}\left\{\frac{m}{\rho_{2}} A^{1 / 2} v+\frac{1}{\rho_{2}} A^{-(\alpha-1 / 2)} B_{1} q\right\} \\
-\frac{1}{\tau} q-\frac{1}{\tau} B_{2} \theta
\end{array}\right)
$$

with domain

$$
\begin{aligned}
D\left(\mathcal{A}_{\alpha, \beta, \gamma, \tau}\right)= & \left\{(u, v, \theta, q)^{T} \in \mathcal{H} \mid v \in D\left(A^{1 / 2}\right), \sigma A^{1-\alpha} u-m \theta \in D\left(A^{\alpha-\gamma / 2}\right), \theta \in D\left(B_{2}\right),\right. \\
& \left.A^{-(\alpha-1 / 2)} B_{1} q \in H, A^{\alpha-1 / 2}\left(\frac{m}{\rho_{2}} A^{1 / 2} v+\frac{1}{\rho_{2}} A^{-(\alpha-1 / 2)} B_{1} q\right) \in H\right\} .
\end{aligned}
$$

Note that $\mathcal{A}_{\alpha, \beta, \gamma, \tau}$ is dissipative, cf. (4.25), and the density of $D\left(\mathcal{A}_{\alpha, \beta, \gamma, \tau}\right)$ in $\mathcal{H}$ is a consequence of the density of $D(A)$ in $H$. Therefore, it is sufficient to prove that $0 \in \rho\left(\mathcal{A}_{\alpha, \beta, \gamma, \tau}\right)$. Analogously to Section 2, we will do this in detail for the parameter region $\beta \geq 2 \alpha-1$. In fact, let us solve the problem

$$
\mathcal{A}_{\alpha, \beta, \gamma, \tau} U=F \equiv\left(f_{1}, f_{2}, f_{3}, f_{4}\right)^{T} \text { in } \mathcal{H} \text {. }
$$


First, let $\alpha \leq 1 / 2$ : Then, defining

$$
\begin{aligned}
v:=f_{1}, \quad \theta:= & -A^{-\beta}\left(\frac{\rho_{2}}{\kappa} f_{3}+\frac{m}{\kappa} A^{\alpha} f_{1}\right)+\frac{\tau}{\kappa} A^{-\beta} B_{1} f_{4} \in D\left(A^{\beta / 2}\right), \quad q:=-B_{2} \theta-\tau f_{4}, \\
u & :=A^{-(1-\alpha)}\left(\frac{m}{\sigma} \theta+\frac{1}{\sigma} A^{-(\alpha-\gamma / 2)}\left(\rho_{1} A^{-\gamma / 2} f_{2}+\mu A^{\gamma / 2} f_{2}\right)\right),
\end{aligned}
$$

we have $(u, v, \theta, q)^{T} \in D\left(\mathcal{A}_{\alpha, \beta, \gamma, \tau}\right)$ and, using (4.23), the resolvent equation and the previous definition of $q$, we have

$$
\|q\|^{2}=\left|\kappa\left(\theta, B_{1} q\right)+\tau\left(f_{4}, q\right)\right| \leq\left|\kappa\left(\theta, m A^{\alpha} f_{1}+\rho_{2} f_{3}\right)\right|+\left|\tau\left(f_{4}, q\right)\right| \leq C|| U\left\|_{\mathcal{H}}\right\| F \|_{\mathcal{H}},
$$

which implies, using again the definition of $q$ and (4.24)

$$
\|\theta\|^{2} \leq C\left\|A^{\beta / 2} \theta\right\|^{2}=C\left\|B_{2} \theta\right\|^{2} \leq C\|q\|^{2}+C\left\|f_{4}\right\|^{2} \leq C\|U\|_{\mathcal{H}}\|F\|_{\mathcal{H}}+C\left\|f_{4}\right\|^{2} .
$$

Combining these estimates with

$$
\begin{gathered}
\|v\|_{D\left(A^{\gamma / 2}\right)} \leq c_{1}\left\|f_{1}\right\|_{D\left(A^{1 / 2}\right)}, \\
\|u\|_{D\left(A^{1 / 2}\right)} \leq c_{1}\left\|A^{-(1 / 2-\alpha)} \theta\right\|+c_{1}\left\|\rho A^{-\gamma / 2} f_{2}+\mu A^{\gamma / 2} f_{2}\right\| \leq c_{1}\left(\|\theta\|+\left\|f_{2}\right\|_{D\left(A^{\gamma / 2}\right)}\right),
\end{gathered}
$$

we obtain the desired inequality $\|U\|_{\mathcal{H}} \leq c_{1}\|F\|_{\mathcal{H}}$.

Now, let $\alpha>1 / 2$ : Then, defining

$$
\begin{aligned}
v:=f_{1}, \quad \theta & :=-A^{-(\beta-(\alpha-1 / 2))}\left(\frac{\rho_{2}}{\kappa} A^{-(\alpha-1 / 2)} f_{3}+\frac{m}{\kappa} A^{1 / 2} f_{1}\right), \quad q:=-B_{2} \theta-\tau f_{4}, \\
u & :=A^{-(1-\alpha)}\left(\frac{m}{\sigma} \theta+\frac{1}{\sigma} A^{-(\alpha-\gamma / 2)}\left(\rho_{1} A^{-\gamma / 2} f_{2}+\mu A^{\gamma / 2} f_{2}\right)\right)
\end{aligned}
$$

we have $(u, v, \theta, q)^{T} \in D\left(\mathcal{A}_{\alpha, \beta, \gamma, \tau}\right)$, where $\theta \in D\left(B_{2}\right)=D\left(A^{\beta / 2}\right)$ because $\beta \geq 2 \alpha-1$. Similarly as in the previous case, working with the definition of $q$, the resolvent equation and the inequality $\beta \geq 2 \alpha-1$, we deduce

$$
\begin{aligned}
\|q\|^{2} & =\left|-\kappa\left(A^{(\alpha-1 / 2)} \theta, A^{-(\alpha-1 / 2)} B_{1} q\right)+\left(f_{4}, q\right)\right| \\
& =\left|\kappa\left(A^{(\alpha-1 / 2)} \theta, m A^{1 / 2} f_{1}+\rho_{2} A^{-(\alpha-1 / 2)} f_{3}\right)+\left(f_{4}, q\right)\right|,
\end{aligned}
$$

which implies

$$
\|q\|^{2} \leq C\left\|A^{\beta / 2} \theta\right\|_{H}\|F\|_{\mathcal{H}}+C\|F\|_{\mathcal{H}}^{2}
$$

Moreover,

$$
\left\|A^{\beta / 2} \theta\right\|^{2}=C\left\|B_{2} \theta\right\|^{2} \leq C\|q\|^{2}+C\left\|f_{4}\right\|^{2} \leq C\left\|A^{\beta / 2} \theta\right\|_{H}\|F\|_{\mathcal{H}}+C\left\|f_{4}\right\|^{2},
$$

implying

$$
\left\|A^{\beta / 2} \theta\right\|^{2} \leq C\|F\|_{\mathcal{H}}^{2}
$$


Finally, we combine these estimates with

$$
\begin{gathered}
\|v\|_{D\left(A^{\gamma / 2}\right)} \leq c_{1}\left\|f_{1}\right\|_{D\left(A^{1 / 2}\right)}, \\
\|u\|_{D\left(A^{1 / 2}\right)} \leq c_{1}\left\|A^{-(\beta-(2 \alpha-1))}\right\|_{H \rightarrow H}\left\|A^{\beta-(\alpha-1 / 2)} \theta\right\|+c_{1}\|F\|_{\mathcal{H}} \leq C\left\|A^{\beta / 2} \theta\right\|+c_{1}\|F\|_{\mathcal{H}}
\end{gathered}
$$

where we used the assumption $\beta \geq 2 \alpha-1$. Hence we have again $\|U\|_{\mathcal{H}} \leq c_{1}\|F\|_{\mathcal{H}}$. Consequently, $\mathcal{A}_{\alpha, \beta, \gamma, \tau}$ generates a $C_{0}$-semigroup $\left\{e^{\mathcal{A}_{\alpha, \beta, \gamma, \tau} t}\right\}_{t \geq 0}$ of contractions on $\mathcal{H}$.

Theorem 4.5. In the exceptional set (4.14) exponential stability is kept.

Proof. We prove condition (2.12). Considering the resolvent equation

$$
i \lambda U-\mathcal{A}_{\alpha, \beta, \gamma, \tau} U=F \quad \text { in } \quad \mathcal{H}
$$

where $U=(u, v, \theta, q)^{T}$ and $F=\left(f_{1}, f_{2}, f_{3}, f_{4}\right)^{T}$, we have

$$
\begin{aligned}
& i \lambda u-v=f, \quad \text { in } \quad V_{1 / 2}, \\
& i \lambda \rho_{1} v+i \lambda \mu A^{\gamma} v+A^{\alpha}\left(\sigma A^{1-\alpha} u-m \theta\right)=\rho_{1} f_{2}+\mu A^{\gamma} f_{2}, \quad \text { in } \quad V_{\gamma / 2}^{\prime}, \\
& i \lambda \rho_{2} \theta+T_{3}=\rho_{2} f_{3}, \quad \text { in } \quad H \\
& i \lambda \tau q+q+B_{2} \theta=\tau f_{4}, \quad \text { in }[H]^{r},
\end{aligned}
$$

having used $v \in V_{1 / 2} \subset V_{\gamma / 2}$ and $\left(I+\frac{\mu}{\rho} A^{\gamma}\right): V_{\gamma / 2} \rightarrow V_{\gamma / 2}^{\prime}$ being an isomorphism, and

$$
T_{3}:=A^{\alpha-1 / 2}\left(m A^{1 / 2} v+A^{-(\alpha-1 / 2)} B_{1} q\right),
$$

for $\alpha \geq 1 / 2$.

Now, multiplying the resolvent equation by $U \in D\left(\mathcal{A}_{\alpha, \beta, \gamma, \tau}\right) \subset \mathcal{H}$, we deduce

$$
-\operatorname{Re}\left(\mathcal{A}_{\alpha, \beta, \gamma, \tau} U, U\right)_{\mathcal{H}}=\operatorname{Re}(U, F)_{\mathcal{H}}
$$

where

$$
\begin{aligned}
-\left(\mathcal{A}_{\alpha, \beta, \gamma, \tau} U, U\right)_{\mathcal{H}}= & -\sigma\left(A^{1 / 2} v, A^{1 / 2} u\right)+\left\langle A^{\alpha}\left(\sigma A^{1-\alpha} u-m \theta\right), v\right\rangle_{V_{\gamma / 2}^{\prime} \times V_{\gamma / 2}} \\
& +\left(T_{3}, \theta\right)+\frac{1}{\kappa}\|q\|^{2}+\frac{1}{\kappa}\left(B_{2} \theta, q\right)_{H^{r}}
\end{aligned}
$$

We have for $\alpha \geq 1 / 2$

$$
\left(m A^{1 / 2} v+A^{-(\alpha-1 / 2)} B_{1} q\right) \in V_{\alpha-1 / 2} \subset H
$$

and, using (4.23),

$$
\theta \in V_{\beta / 2}=V_{\alpha-\gamma / 2} \subset V_{\alpha-1 / 2} \subset H, \text { because } \quad \frac{\beta}{2}=\alpha-\frac{\gamma}{2} \quad \text { in } \quad \mathcal{S}_{\text {exc }} .
$$


Then we have

$$
\begin{aligned}
-\left(\mathcal{A}_{\alpha, \beta, \gamma, \tau} U, U\right)_{\mathcal{H}}= & -\sigma\left(A^{1 / 2} v, A^{1 / 2} u\right)+\left\langle A^{\alpha}\left(\sigma A^{1-\alpha} u-m \theta\right), v\right\rangle_{V_{\gamma / 2}^{\prime} \times V_{\gamma / 2}} \\
& +\left(m A^{1 / 2} v+A^{-(\alpha-1 / 2)} B_{1} q, A^{\alpha-1 / 2} \theta\right)+\frac{1}{\kappa}\|q\|^{2}+\frac{1}{\kappa}\left(B_{2} \theta, q\right)_{H^{r}} \\
= & -\left(A^{1 / 2} v, A^{\alpha-1 / 2}\left(\sigma A^{1-\alpha} u-m \theta\right)\right)+\left(A^{\alpha-1 / 2}\left(\sigma A^{1-\alpha} u-m \theta\right), A^{1 / 2} v\right) \\
& -\frac{1}{\kappa}\left(q, B_{2} \theta\right)_{H^{r}}+\frac{1}{\kappa}\|q\|^{2}+\frac{1}{\kappa}\left(B_{2} \theta, q\right)_{H^{r}}
\end{aligned}
$$

which implies

$$
-\operatorname{Re}\left(\mathcal{A}_{\alpha, \beta, \gamma, \tau} U, U\right)_{\mathcal{H}}=\frac{1}{\kappa}\|q\|^{2} .
$$

Then, using (4.34), (4.35), we obtain the first dissipation inequality (corresponding to (4.25))

$$
\|q\|^{2} \leq C\|U\|_{\mathcal{H}}\|F\|_{\mathcal{H}}
$$

Now, let us define further multipliers given by $\psi$ and $\phi$ as the solutions to the equations

$$
A^{\beta} \phi=\theta \quad \text { and } \quad A^{\beta} \psi=B_{1} q
$$

Since

$$
\theta \in D\left(B_{2}\right)=V_{\beta / 2} \subset H \quad \text { and } \quad B_{1} q \in W:=\left\{\begin{array}{ll}
H & \text { for } \alpha=1 / 2 \\
V_{\alpha-1 / 2}^{\prime} & \text { for } \alpha>1 / 2
\end{array},\right.
$$

we deduce

$$
\phi \in V_{\beta} \subset H \quad \text { and } \quad \psi \in V_{\beta-(\alpha-1 / 2)} \subset V_{\beta / 2} \quad \text { in } \quad \mathcal{S}_{\text {exc }}, \quad \text { for } \quad \alpha \geq 1 / 2 \text {. }
$$

Then, using equation (4.32) with $B_{2} \phi \in[H]^{r}$, we have

$$
\underbrace{i \lambda \tau\left(q, B_{2} \phi\right)_{[H]^{r}}}_{=: J_{4}}+\underbrace{\left(q, B_{2} \phi\right)_{[H]^{r}}}_{=: J_{3}}+\underbrace{\left(B_{2} \theta, B_{2} \phi\right)_{[H]^{r}}}_{=: J_{2}}=\underbrace{\tau\left(f_{4}, B_{2} \phi\right)_{[H]^{r}}}_{=: J_{1}}
$$

Using $V_{\beta} \subset D\left(B_{2}\right)$, we have

$$
\left|J_{1}\right| \leq\left\|f_{4}\right\| \cdot\left\|B_{2} \phi\right\| \leq C\left\|f_{4}\right\| \cdot\left\|A^{\beta / 2} \phi\right\| \leq C\|U\|_{\mathcal{H}}\|F\|_{\mathcal{H}} .
$$

Using (4.23) we get

$$
\begin{gathered}
J_{2}=\left(\theta, B_{2}^{*} B_{2} \phi\right)=-\kappa\left(\theta, B_{1} B_{2} \phi\right)=\kappa^{2}\left(\theta, A^{\beta} \phi\right)=\kappa^{2}\|\theta\|^{2}, \\
\left|J_{3}\right|=\left|\left(q, B_{2} \phi\right)\right| \leq C\|q\| \cdot\left\|A^{\beta} \phi\right\| \leq\|q\| \cdot\|\theta\| .
\end{gathered}
$$

Again, using (4.23), we obtain

$$
J_{4}=-i \lambda \tau \kappa\left\langle B_{1} q, \phi\right\rangle_{V_{\alpha-1 / 2}^{\prime} \times V_{\alpha-1 / 2}}
$$


for $\alpha \geq 1 / 2$, because $\phi \in V_{\beta} \subset V_{\alpha-1 / 2}$ in $\mathcal{S}_{\text {exc }}$. Consequently, using (4.31) and the regularity of $\phi$ and $\psi$, we obtain

$$
J_{4}=-i \lambda \tau \kappa(\psi, \theta)=\tau \kappa(\psi, i \lambda \theta)=-\frac{\tau \kappa}{\rho_{2}}\left(\psi, T_{3}\right)+\tau \kappa\left(\psi, f_{3}\right) .
$$

Then, using the definition of $T_{3}$, the regularity $\psi \in V_{\beta / 2}$ and the identity $\alpha=\frac{\gamma}{2}+\frac{\beta}{2}$ in $\mathcal{S}_{\text {exc }}$, this results in

$$
J_{4}=\underbrace{-\frac{\tau \kappa}{\rho_{2}}\left\|A^{\beta / 2} \psi\right\|^{2}}_{=: J_{4}^{1}} \underbrace{-\frac{\tau \kappa m}{\rho_{2}}\left(A^{\beta / 2} \psi, A^{\gamma / 2} v\right)}_{=: J_{4}^{2}}+\underbrace{\tau \kappa\left(\psi, f_{3}\right)}_{=: J_{4}^{3}}
$$

where

$$
\begin{aligned}
&\left|J_{4}^{1}\right|=\frac{\tau \kappa}{\rho_{2}}\left\|A^{\beta / 2} \psi\right\|^{2} \leq C\|q\|^{2}, \\
&\left|J_{4}^{2}\right|=C\left|\left(A^{\beta / 2} \psi, A^{\gamma / 2} v\right)\right| \leq C\|q\| \cdot\left\|A^{\gamma / 2} v\right\|, \\
&\left|J_{4}^{3}\right| \leq C\|\psi\| \cdot\left\|f_{3}\right\| \leq C\|U\|_{\mathcal{H}}\|F\|_{\mathcal{H}},
\end{aligned}
$$

which implies

$$
\left|J_{4}\right| \leq C|| U\left\|_{\mathcal{H}}\right\| F\left\|_{\mathcal{H}}+C\right\| q\|\cdot\| A^{\gamma / 2} v \| .
$$

Therefore, substituting into (4.38) and using (4.36) we have

$$
\|\theta\|^{2} \leq C\|U\|_{\mathcal{H}}\|F\|_{\mathcal{H}}+C_{1}\|q\| \cdot\left\|A^{\gamma / 2} v\right\|
$$

On the other hand, using equation (4.30) and $u \in V_{1 / 2} \hookrightarrow V_{\gamma / 2}$, we have

$$
\left\langle i \lambda \rho_{1} v+i \lambda \mu A^{\gamma} v, u\right\rangle_{V_{\gamma / 2}^{\prime} \times V_{\gamma / 2}}+\left\langle A^{\alpha}\left(\sigma A^{1-\alpha} u-m \theta\right), u\right\rangle_{V_{\gamma / 2}^{\prime} \times V_{\gamma / 2}}=\rho_{1}\left\langle f_{2}+\mu A^{\gamma} f_{2}, u\right\rangle_{V_{\gamma / 2}^{\prime} \times V_{\gamma / 2}} .
$$

Then, using equation (4.29), we have

$$
\left\langle\rho_{1} v+\mu A^{\gamma} v,-v-f_{1}\right\rangle_{V_{\gamma / 2}^{\prime} \times V_{\gamma / 2}}+\left\langle A^{\alpha}\left(\sigma A^{1-\alpha} u-m \theta\right), u\right\rangle_{V_{\gamma / 2}^{\prime} \times V_{\gamma / 2}}=\rho_{1}\left\langle f_{2}+\mu A^{\gamma} f_{2}, u\right\rangle_{V_{\gamma / 2}^{\prime} \times V_{\gamma / 2}}
$$

which implies, for $v \in V_{1 / 2} \hookrightarrow V_{\gamma / 2}$,

$$
\begin{aligned}
\underbrace{\left\langle A^{\alpha}\left(\sigma A^{1-\alpha} u-m \theta\right), u\right\rangle_{V_{\gamma / 2}^{\prime} \times V_{\gamma / 2}}=}_{=: J_{7}} & \rho_{1}\left(v, f_{1}\right)+\mu\left\|A^{\gamma / 2} v\right\|^{2}+\rho_{1}\|v\|^{2}+\underbrace{\mu\left\langle A^{\gamma} v, f_{1}\right\rangle_{V_{\gamma / 2}^{\prime} \times V_{\gamma / 2}}}_{=: J_{5}} \\
& +\underbrace{\rho_{1}\left\langle f_{2}+\mu A^{\gamma} f_{2}, u\right\rangle_{V_{\gamma / 2}^{\prime} \times V_{\gamma / 2}}}_{=: J_{6}} .
\end{aligned}
$$

Note that

$$
\left|J_{5}\right|=\mu\left|\left(A^{\gamma / 2} v, A^{\gamma / 2} f_{1}\right)\right| \leq C|| A^{\gamma / 2} v\|\| A^{\gamma / 2} f_{1}\left\|\leq C_{1}\right\| A^{\gamma / 2} v\|\| A^{1 / 2} f_{1}\|\leq C\| U\left\|_{\mathcal{H}}\right\| F \|_{\mathcal{H}} .
$$


Similarly we have

$$
\begin{aligned}
\left|J_{6}\right| \leq \rho_{1}\left|\left(f_{2}, u\right)\right|+\rho_{1} \mu\left|\left(A^{\gamma / 2} f_{2}, A^{\gamma / 2} u\right)\right| & \leq C\|U\|_{\mathcal{H}}\|F\|_{\mathcal{H}}+C\left\|A^{\gamma / 2} f_{2}\right\|\left\|A^{\gamma / 2} u\right\| \\
& \leq C\|U\|_{\mathcal{H}}\|F\|_{\mathcal{H}} .
\end{aligned}
$$

For $\alpha \geq 1 / 2,\left(\sigma A^{1-\alpha} u-m \theta\right) \in V_{\alpha-\gamma / 2}$ and using again $\alpha=\frac{\beta}{2}+\frac{\gamma}{2}$ in $\mathcal{S}_{\text {exc }}$, we obtain

$$
J_{7}=\left(A^{\alpha-\gamma / 2}\left(\sigma A^{1-\alpha} u-m \theta\right), A^{\gamma / 2} u\right)=\sigma\left\|A^{1 / 2} u\right\|^{2}-m\left(A^{\beta / 2} \theta, A^{\gamma / 2} u\right) .
$$

Therefore, using (4.41)-(4.43) in (4.40) we obtain

$$
\sigma\left\|A^{1 / 2} u\right\|^{2} \leq C\|U\|_{\mathcal{H}}\|F\|_{\mathcal{H}}+\rho_{1}\|v\|^{2}+\mu\left\|A^{\gamma / 2} v\right\|^{2}+m\left|\left(A^{\beta / 2} \theta, A^{\gamma / 2} u\right)\right| .
$$

Here, using (4.29) we obtain

$m\left|\left(A^{\beta / 2} \theta, A^{\gamma / 2} u\right)\right|=m\left|\left(A^{\beta / 2} \theta, \frac{A^{\gamma / 2} v+A^{\gamma / 2} f_{1}}{i \lambda}\right)\right| \leq \frac{m}{|\lambda|}\left|\left(A^{\beta / 2} \theta, A^{\gamma / 2} v\right)\right|+\frac{m}{|\lambda|}\left|\left(A^{\beta / 2} \theta, A^{\gamma / 2} f_{1}\right)\right|$,

which implies, using (4.32),

$$
\begin{aligned}
m\left|\left(A^{\beta / 2} \theta, A^{\gamma / 2} u\right)\right| & \leq \frac{m}{|\lambda|}\left\|A^{\beta / 2} \theta\right\| \cdot\left\|A^{\gamma / 2} v\right\|+\frac{m}{|\lambda|}\left\|A^{\beta / 2} \theta\right\| \cdot\left\|A^{\gamma / 2} f_{1}\right\| \\
& \leq \frac{C_{1}}{|\lambda|}\left\|B_{2} \theta\right\| \cdot\left\|A^{\gamma / 2} v\right\|+\frac{C_{2}}{|\lambda|}\left\|B_{2} \theta\right\| \cdot\left\|A^{\gamma / 2} f_{1}\right\| \\
& \leq \frac{C_{1}}{|\lambda|}\left(|\lambda|\|q\|+\|q\|+\left\|f_{4}\right\|\right)\left(\left\|A^{\gamma / 2} v\right\|+\left\|A^{\gamma / 2} f_{1}\right\|\right) \\
& \leq C_{1}\|q\| \cdot\left\|A^{\gamma / 2} v\right\|+C_{1}\|U\|_{\mathcal{H}}\|F\|_{\mathcal{H}}+\frac{C_{2}}{|\lambda|}\|F\|_{\mathcal{H}}^{2},
\end{aligned}
$$

for $|\lambda| \geq 1$. Therefore substituting this into (4.44) and using (4.36) we obtain

$$
\sigma\left\|A^{1 / 2} u\right\|^{2} \leq C\|U\|_{\mathcal{H}}\|F\|_{\mathcal{H}}+\left[\rho_{1}\|v\|^{2}+\mu\left\|A^{\gamma / 2} v\right\|^{2}\right]+C_{1}\|q\| \cdot\left\|A^{\gamma / 2} v\right\|+\frac{C_{2}}{|\lambda|}\|F\|_{\mathcal{H}}^{2} .
$$

Now, in order to estimate $\rho_{1}\|v\|^{2}+\mu\left\|A^{\gamma / 2} v\right\|^{2}$, let us define new multipliers $y$ and $z$ as the solutions to the equations

$$
A^{\alpha} y=\theta \quad \text { and } \quad A^{\alpha} z=v
$$

Since

$$
\theta \in D\left(B_{2}\right) \subset H \quad \text { and } \quad v \in V_{1 / 2}
$$

we deduce

$$
y \in V_{\alpha} \subset H \quad \text { and } \quad z \in V_{\alpha+1 / 2} .
$$

Then, with equation (4.30), we have for $y \in V_{\alpha} \hookrightarrow V_{\gamma / 2}$

$$
\begin{aligned}
\underbrace{i \lambda \rho_{1}(v, y)}_{=: J_{8}}+ & \underbrace{i \lambda \mu\left\langle A^{\gamma} v, y\right\rangle_{V_{\gamma / 2}^{\prime} \times V_{\gamma / 2}}}_{=: J_{9}}+ \\
& \left\langle A^{\alpha}\left(\sigma A^{1-\alpha} u-m \theta\right), y\right\rangle_{V_{\gamma / 2}^{\prime} \times V_{\gamma / 2}}=\rho_{1}\left(f_{2}, y\right)+\mu\left\langle A^{\gamma} f_{2}, y\right\rangle_{V_{\gamma / 2}^{\prime} \times V_{\gamma / 2}} .
\end{aligned}
$$


Using (4.46) and equation (4.31), we get

$$
\begin{aligned}
J_{8} & =i \lambda \rho_{1}\left(A^{\alpha} z, y\right)=i \lambda \rho_{1}\left(z, A^{\alpha} y\right) \\
& =i \lambda \rho_{1}(z, \theta)=\rho_{1}(z,-i \lambda \theta) \\
& =\frac{\rho_{1}}{\rho_{2}}\left(z, T_{3}-\rho_{2} f_{3}\right),
\end{aligned}
$$

which implies, for $\alpha \geq 1 / 2$, using the regularity of $z$,

$$
\begin{aligned}
J_{8} & =\frac{\rho_{1}}{\rho_{2}}\left(z, A^{\alpha-1 / 2}\left(m A^{1 / 2} v+A^{-(\alpha-1 / 2)} B_{1} q\right)-\rho_{2} f_{3}\right) \\
& =\frac{\rho_{1}}{\rho_{2}}\left(A^{\alpha-1 / 2} z, m A^{1 / 2} v+A^{-(\alpha-1 / 2)} B_{1} q\right)-\rho_{2}\left(z, f_{3}\right) \\
& =\frac{m \rho_{1}}{\rho_{2}}\|v\|^{2}-\frac{\rho_{1}}{\rho_{2} \kappa}\left(B_{2} z, q\right)-\rho_{1}\left(z, f_{3}\right) .
\end{aligned}
$$

Consequently

$$
J_{8}=-\frac{\rho_{1}}{\rho_{2} \kappa}\left(B_{2} z, q\right)+\frac{m \rho_{1}}{\rho_{2}}\|v\|^{2}-\rho_{1}\left(z, f_{3}\right) .
$$

Moreover, using $v \in V_{\gamma / 2}$ and equations (4.46), (4.31) we have

$$
\begin{aligned}
J_{9} & =i \lambda \mu\left(A^{\gamma / 2} v, A^{\gamma / 2} y\right)=\mu\left(A^{\gamma / 2+\alpha} z,-i \lambda A^{\gamma / 2} y\right)=\mu\left(A^{\gamma} z,-i \lambda \theta\right) \\
& =\frac{\mu}{\rho_{2}}\left(A^{\gamma} z, T_{3}-\rho_{2} f_{3}\right)
\end{aligned}
$$

which implies, for $\alpha \geq 1 / 2$,

$$
\begin{aligned}
J_{9} & =\frac{\mu}{\rho_{2}}\left(A^{\gamma} z, A^{\alpha-1 / 2}\left(m A^{1 / 2} v+A^{-(\alpha-1 / 2)} B_{1} q\right)-\rho_{2} f_{3}\right) \\
& =\frac{\mu}{\rho_{2}}\left(A^{\gamma+\alpha-1 / 2} z, m A^{1 / 2} v+A^{-(\alpha-1 / 2)} B_{1} q\right)-\mu\left(A^{\gamma} z, f_{3}\right) \\
& =\frac{\mu m}{\rho_{2}}\left\|A^{\gamma / 2} v\right\|^{2}+\frac{\mu}{\rho_{2}}\left(A^{\gamma+\alpha-1 / 2} z, A^{-(\alpha-1 / 2)} B_{1} q\right)-\mu\left(A^{\gamma} z, f_{3}\right) .
\end{aligned}
$$

Then, substituting this into (4.47) yields

$$
\begin{aligned}
-\frac{\rho_{1}}{\rho_{2} \kappa}\left(B_{2} z, q\right) & +\frac{m \rho_{1}}{\rho_{2}}\|v\|^{2}-\rho_{1}\left(z, f_{3}\right)+\frac{\mu}{\rho_{2}} T_{4}+\frac{\mu m}{\rho_{2}}\left\|A^{\gamma / 2} v\right\|^{2}-\mu\left(A^{\gamma} z, f_{3}\right)+ \\
& +\left\langle A^{\alpha}\left(\sigma A^{1-\alpha} u-m \theta\right), y\right\rangle_{V_{\gamma / 2}^{\prime} \times V_{\gamma / 2}}=\rho_{1}\left(f_{2}, y\right)+\mu\left\langle A^{\gamma} f_{2}, y\right\rangle_{V_{\gamma / 2}^{\prime} \times V_{\gamma / 2}},
\end{aligned}
$$

where

$$
T_{4}:=\left(A^{\gamma+\alpha-1 / 2} z, A^{-(\alpha-1 / 2)} B_{1} q\right) \quad \text { for } \quad \alpha \geq \frac{1}{2} .
$$

Here, using $\frac{\beta}{2} \leq \alpha$, and condition (4.23) we get

$$
\left|\left(B_{2} z, q\right)\right| \leq\left\|A^{\beta / 2} z|\|q\| \leq C|\left|A^{\alpha} z\right|\right\| q\|=C\| v\|\| q \| .
$$


Using again $\gamma+\beta=2 \alpha$ we deduce, for $\alpha \geq 1 / 2$

$$
\begin{aligned}
\left|T_{4}\right|=\left(A^{\gamma+\alpha-1 / 2} z, A^{-(\alpha-1 / 2)} B_{1} q\right) & =\left|\left(A^{\gamma-1 / 2} v, A^{-(\alpha-1 / 2)} B_{1} q\right)\right|=\frac{1}{\kappa}\left|\left(B_{2} A^{\gamma / 2-\alpha} A^{\gamma / 2} v, q\right)\right| \\
& \leq C\left\|B_{2} A^{\gamma / 2-\alpha} A^{\gamma / 2} v\right\| \cdot\|q\| \leq C\left\|A^{\beta / 2+\gamma / 2-\alpha} A^{\gamma / 2} v\right\| \cdot\|q\| \\
& =C\left\|A^{\gamma / 2} v\right\| \cdot\|q\| .
\end{aligned}
$$

Also

$\left|\left(A^{\gamma} z, f_{3}\right)\right| \leq\left|\left(A^{\gamma / 2-\alpha} A^{\gamma / 2} v, f_{3}\right)\right| \leq\left\|A^{-\beta / 2} A^{\gamma / 2} v\right\| \cdot\left\|f_{3}\right\| \leq C\left\|A^{\gamma / 2} v\right\| \cdot\left\|f_{3}\right\| \leq C\|U\|_{\mathcal{H}}\|F\|_{\mathcal{H}}$,

and, using $\alpha \geq \frac{1}{2}$,

$$
\begin{aligned}
\left|\left\langle A^{\alpha}\left(\sigma A^{1-\alpha} u-m \theta\right), y\right\rangle_{V_{\gamma / 2}^{\prime} \times V_{\gamma / 2}}\right| & =\left|\left(\sigma A^{1-\alpha} u-m \theta, A^{\alpha} y\right)\right| \\
& \leq C\left\|A^{1-\alpha} u\right\| \cdot\|\theta\|+m\|\theta\|^{2} \\
& \leq C\left\|A^{1 / 2} u\right\| \cdot\|\theta\|+m\|\theta\|^{2},
\end{aligned}
$$

and, using $\frac{\gamma}{2} \leq \alpha$,

$$
\begin{aligned}
\mid\left\langle A^{\gamma} f_{2}, y\right\rangle_{V_{\gamma / 2}^{\prime} \times V_{\gamma / 2} \mid} \leq C\left\|A^{\gamma / 2} f_{2}\right\| \cdot\left\|A^{\gamma / 2} y\right\| \\
\leq C\left\|A^{\gamma / 2} f_{2}\right\| \cdot\left\|A^{\alpha} y\right\| \leq C\left\|A^{\gamma / 2} f_{2}\right\| \cdot\|\theta\| \leq C\|U\|_{\mathcal{H}}\|F\|_{\mathcal{H}} .
\end{aligned}
$$

Inserting all into (4.48) we obtain

$$
\rho_{1}\|v\|^{2}+\mu\left\|A^{\gamma / 2} v\right\|^{2} \leq C\|U\|_{\mathcal{H}}\|F\|_{\mathcal{H}}+C\left\|A^{1 / 2} u\right\| \cdot\|\theta\|+C\|\theta\|^{2} .
$$

Finally, we combine all the results to get exponential stability. In fact, taking $2 \times(4.49)+$ (4.45) and using (4.36) we have

$$
\frac{1}{2}\left[\sigma\left\|A^{1 / 2} u\right\|^{2}+\rho_{1}\|v\|^{2}+\mu\left\|A^{\gamma / 2} v\right\|^{2}\right] \leq C\|U\|_{\mathcal{H}}\|F\|_{\mathcal{H}}+\frac{C_{2}}{|\lambda|}\|F\|_{\mathcal{H}}^{2}+C_{0}\|\theta\|^{2},
$$

for $|\lambda| \geq 1$. Now, taking $2 C_{0} \times(4.39)+(4.50)$ and using (4.36) yields

$$
\frac{1}{4}\left[\sigma\left\|A^{1 / 2} u\right\|^{2}+\rho_{1}\|v\|^{2}+\mu\left\|A^{\gamma / 2} v\right\|^{2}\right]+C_{0}\|\theta\|^{2} \leq C\|U\|_{\mathcal{H}}\|F\|_{\mathcal{H}}+\frac{C_{2}}{|\lambda|}\|F\|_{\mathcal{H}}^{2} .
$$

So, combining (4.51) with (4.36) we deduce

$$
\|U\|_{\mathcal{H}}^{2} \leq C\|U\|_{\mathcal{H}}\|F\|_{\mathcal{H}}+\frac{C_{2}}{|\lambda|}\|F\|_{\mathcal{H}}^{2},
$$

which implies

$$
\|U\|_{\mathcal{H}} \leq C\|F\|_{\mathcal{H}},
$$

with $C$ being independent of $\lambda$, which proves Theorem 4.5. 


\section{Polynomial stability}

In this section, we will study the polynomial stability of the semigroup $\left\{e^{\mathcal{A}_{\alpha, \beta, \gamma} t}\right\}_{t \geq 0}$ for $(\alpha, \beta, \gamma)$, case $\tau=0,0<\gamma<1$, in the subset $S_{2}$, see Figure 5.1. In the region $S_{3}$ or for $\gamma=1$ in $S_{2}$, purely imaginary points are expected in the spectrum, see the remarks at the end.

Define

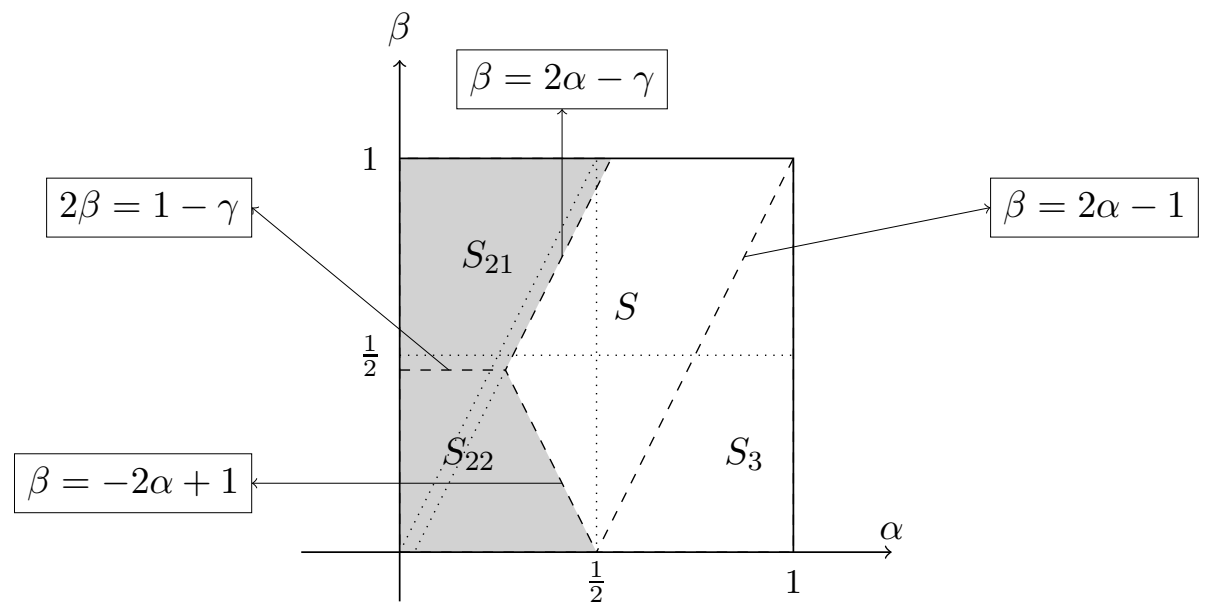

Figure 5.1: Regions of polynomial stability for Fourier.

$$
S_{21}:=\left\{(\alpha, \beta, \gamma) \in[0,1] \times[0,1] \times(0,1): 0 \leq \alpha<\frac{\beta+\gamma}{2}, \frac{1-\gamma}{2} \leq \beta \leq 1\right\}
$$

and

$$
S_{22}:=\left\{(\alpha, \beta, \gamma) \in[0,1] \times[0,1] \times(0,1): 0 \leq \alpha<\frac{1-\beta}{2}, 0 \leq \beta<\frac{1-\gamma}{2}\right\} .
$$

Our tool is the following frequency domain characterization of polynomial stability by Borichev and Tomilov [3].

Theorem 5.1. Let $\mathcal{H}$ be a Hilbert space and let $\mathcal{A}$ generate a bounded $C_{0}$-semigroup in $\mathcal{H}$. Assume that

$$
\begin{gathered}
i \mathbb{R} \subset \rho(\mathcal{A}), \\
\limsup _{\lambda \in \mathbb{R},|\lambda|>1} \frac{1}{|\lambda|^{p}}\left\|(i \beta-\mathcal{A})^{-1}\right\|_{\mathcal{L}(H)}<\infty, \quad \text { for some } p>0 .
\end{gathered}
$$

Then, there exists a positive constant $C>0$ such that for all $t>0$ we have

$$
\left\|e^{\mathcal{A} t} U_{0}\right\| \leq C\left(\frac{1}{t}\right)^{\frac{1}{p}}\left\|U_{0}\right\|_{D(\mathcal{A})},
$$

for all $U_{0} \in D(\mathcal{A})$.

The polynomial decay in the region $S_{2}$ is described in the following theorem. 
Theorem 5.2. (i). For $(\alpha, \beta, \gamma) \in S_{21}$, there exists a constant $C>0$ such that

$$
\left\|e^{\mathcal{A}_{\alpha, \beta, \gamma} t} U_{0}\right\| \leq \frac{C}{t^{\frac{1-\gamma}{2(\beta-2 \alpha+\gamma)}}}\left\|U_{0}\right\|_{D\left(\mathcal{A}_{\alpha, \beta, \gamma}\right)}
$$

for all $t>0$ and for all $U_{0} \in D\left(\mathcal{A}_{\alpha, \beta, \gamma}\right)$.

(ii). For $(\alpha, \beta, \gamma) \in S_{22}, g$ there exists a constant $C>0$ such that

$$
\left\|e^{\mathcal{A}_{\alpha, \beta, \gamma} t} U_{0}\right\| \leq \frac{C}{t^{\frac{1-\gamma}{2(1-2 \alpha-\beta)}}}\left\|U_{0}\right\|_{D\left(\mathcal{A}_{\alpha, \beta, \gamma}\right)}
$$

for all $t>0$ and for all $U_{0} \in D\left(\mathcal{A}_{\alpha, \beta, \gamma}\right)$.

We shall prove this theorem by verifying conditions (5.3), (5.4) in the following three lemmas.

Lemma 5.3. Let $p:=\frac{2(\beta-2 \alpha+\gamma)}{1-\gamma}$. Then

$$
\limsup _{\lambda \in \mathbb{R},|\lambda| \rightarrow \infty} \frac{1}{\left|\lambda^{p}\right|}\left\|\left(i \lambda-\mathcal{A}_{\alpha, \beta, \gamma}\right)^{-1}\right\|_{\mathcal{L}(\mathcal{H})}<\infty
$$

for $(\alpha, \beta, \gamma) \in S_{21}$

Proof: Assume that (5.6) is false. Then, there exist a sequence $\lambda_{n} \rightarrow \infty$ ( $+\infty$ w.l.o.g.) and a unit sequence $U_{n}=\left(u_{n}, v_{n}, \theta_{n}\right) \in D\left(\mathcal{A}_{\alpha, \beta, \gamma}\right)$ such that $\lambda_{n}^{p}\left(i \lambda_{n} I-\mathcal{A}\right) U_{n}=F_{n} \equiv\left(f_{n 1}, f_{n 2}, f_{n 3}\right)$ and

$$
\lambda_{n}^{p}\left\|\left(i \lambda_{n} I-\mathcal{A}\right) U_{n}\right\|_{\mathcal{H}}=\left\|F_{n}\right\|_{\mathcal{H}}=o(1)
$$

i.e.,

$$
\begin{array}{r}
\lambda_{n}^{p}\left(i \lambda_{n} u_{n}-v_{n}\right)=f_{1 n}=o(1), \text { in } V_{1 / 2}, \\
\lambda_{n}^{p}\left(i \lambda_{n}\left(\rho v_{n}+\mu A^{\gamma} v_{n}\right)+\sigma A u_{n}-m A^{\alpha} \theta_{n}\right)=\rho f_{2 n}+\mu A^{\gamma} f_{2 n}=o(1), \text { in } V_{\gamma / 2}^{\prime}, \\
\lambda_{n}^{p}\left(i \lambda_{n} c \theta_{n}+A^{\alpha-1 / 2}\left[k A^{\beta-(\alpha-1 / 2)} \theta_{n}+m A^{1 / 2} v_{n}\right]\right)=c f_{3 n}=o(1), \text { in } H .
\end{array}
$$

We are going to show $\left\|U_{n}\right\|_{\mathcal{H}}=o(1)$ which is a contradiction. It follows from

$$
\Re\left\langle\mathcal{A}_{\alpha, \beta, \gamma} U_{n}, U_{n}\right\rangle_{\mathcal{H}}=-k\left\|A^{\frac{\beta}{2}} \theta_{n}\right\|^{2}
$$

and (5.7) that

$$
\lambda_{n}^{\frac{p}{2}}\left\|A^{\frac{\beta}{2}} \theta_{n}\right\|=o(1) \Rightarrow\left\|\theta_{n}\right\|=o(1) .
$$

Note that $p>0$ in $S_{21}$ since $\alpha<\frac{\beta+\gamma}{2}$. Acting the bounded operator $\lambda_{n}^{-p-1} A^{-\frac{\gamma}{2}}$ on (5.9) yields

$$
i \rho A^{-\frac{\gamma}{2}} v_{n}+i \mu A^{\frac{\gamma}{2}} v_{n}+\lambda_{n}^{-1} A^{\alpha-\frac{\gamma}{2}}\left(\sigma A^{1-\alpha} u_{n}-m \theta_{n}\right)=o(1), \quad \text { in } \quad H .
$$

The first two terms in (5.13) are bounded in $H$. Thus,

$$
\left\|\lambda_{n}^{-1} A^{\alpha-\frac{\gamma}{2}}\left(\sigma A^{1-\alpha} u_{n}-m \theta_{n}\right)\right\|=O(1) .
$$


Next, acting the bounded operator $\lambda_{n}^{-\frac{p}{2}} A^{-\frac{\beta}{2}}$ on (5.10) yields, using (5.12),

$$
i \lambda_{n}^{\frac{p}{2}+1} c A^{-\frac{\beta}{2}} \theta_{n}+i \lambda_{n}^{\frac{p}{2}+1} m A^{\alpha-\frac{\beta}{2}} u_{n}=o(1) \text { in } H,
$$

where the third term is removed due to (5.12) and $\alpha-\frac{1}{2}<\frac{\beta}{2}$ in $S_{21} . v_{n}$ in the second term is replaced by $\lambda_{n} u_{n}$ validated by (5.8) and the property $\alpha-\frac{\beta}{2}<\frac{\gamma}{2}$ in $S_{21}$. Taking the inner product of (5.15) with $\lambda_{n}^{-1} A^{\alpha-\frac{\gamma}{2}}\left(\sigma A^{1-\alpha} u_{n}-m \theta_{n}\right)$ in $H$, we have

$$
\begin{aligned}
o(1)= & \left(c \lambda_{n}^{\frac{p}{2}+1} A^{-\frac{\beta}{2}} \theta_{n}+m \lambda_{n}^{\frac{p}{2}+1} A^{\alpha-\frac{\beta}{2}} u_{n}, \lambda_{n}^{-1} A^{\alpha-\frac{\gamma}{2}}\left(\sigma A^{1-\alpha} u_{n}-m \theta_{n}\right)\right) \\
= & \left(c \lambda_{n}^{\frac{p}{2}} A^{\alpha-\frac{\gamma}{2}-\frac{\beta}{2}} \theta_{n}+m \lambda_{n}^{\frac{p}{2}} A^{2 \alpha-\frac{\gamma}{2}-\frac{\beta}{2}} u_{n}, \sigma A^{1-\alpha} u_{n}-m \theta_{n}\right) \\
= & c \sigma\left(\lambda_{n}^{\frac{p}{2}} A^{\frac{\beta}{2}} \theta_{n}, A^{1-\frac{\gamma}{2}-\beta} u_{n}\right)-c m\left\|\lambda_{n}^{\frac{p}{4}} A^{\frac{2 \alpha-\gamma-\beta}{4}} \theta_{n}\right\|^{2}+m \sigma\left\|\lambda_{n}^{\frac{p}{4}} A^{\frac{2-\beta+2 \alpha-\gamma}{4}} u_{n}\right\|^{2} \\
& -m^{2}\left(A^{2 \alpha-\frac{\gamma}{2}-\beta} u_{n}, \lambda_{n}^{\frac{p}{2}} A^{\frac{\beta}{2}} \theta_{n}\right) .
\end{aligned}
$$

Note that in $S_{21}$, we have

$$
\left\{\begin{array}{l}
\alpha-\frac{\gamma}{2}-\frac{\beta}{2}<0 \\
2 \alpha-\frac{\gamma}{2}-\beta \leq 1-\alpha \\
1-\frac{\gamma}{2}-\beta \leq \frac{1}{2} \\
\frac{2-\beta+2 \alpha-\gamma}{4}<\frac{1}{2} \\
2 \alpha-\frac{\gamma}{2}-\beta<\frac{1}{2}
\end{array}\right.
$$

Thus, all the terms in (5.16) are well defined. Since $\lambda_{n}^{\frac{p}{4}}\left\|A^{\frac{2 \alpha-\gamma-\beta}{4}} \theta_{n}\right\|=o(1)$ due to (5.12), and

$$
\left\{\begin{array}{l}
\left|\left(\lambda_{n}^{\frac{p}{2}} A^{\frac{\beta}{2}} \theta_{n}, A^{1-\frac{\gamma}{2}-\beta} u_{n}\right)\right| \leq\left\|\lambda_{n}^{\frac{p}{2}} A^{\frac{\beta}{2}} \theta_{n}\right\|\left\|A^{\frac{1}{2}} u_{n}\right\|=o(1) \\
\left|\left(A^{2 \alpha-\frac{\gamma}{2}-\beta} u_{n}, \lambda_{n}^{\frac{p}{2}} A^{\frac{\beta}{2}} \theta_{n}\right)\right| \leq\left\|\lambda_{n}^{\frac{p}{2}} A^{\frac{\beta}{2}} \theta_{n}\right\|\left\|A^{\frac{1}{2}} u_{n}\right\|=o(1),
\end{array}\right.
$$

it follows from (5.16) that

$$
\left\|\lambda_{n}^{\frac{p}{4}} A^{\frac{2-\beta+2 \alpha-\gamma}{4}} u_{n}\right\|=o(1)
$$

which further leads to

$$
\left\|\lambda_{n}^{\frac{p}{4}} A^{\frac{2-\beta+2 \alpha-\gamma}{4}-1+\alpha}\left(\sigma A^{1-\alpha} u_{n}-m \theta_{n}\right)\right\|=o(1),
$$

On the other hand, note that

$$
\frac{2-\beta+2 \alpha-\gamma}{4}-1+\alpha<\alpha-\frac{1}{2}<\alpha-\frac{\gamma}{2}
$$

because $\gamma<1$ and $\beta-2 \alpha+\gamma>0$ in $S_{21}$. By interpolation (cf. [23]) we obtain

$$
\begin{aligned}
\left\|A^{\alpha-\frac{1}{2}} y_{n}\right\| & \leq C\left\|A^{\alpha-\frac{\gamma}{2}} y_{n}\right\|^{a}\left\|A^{\frac{2-\beta+2 \alpha-\gamma}{4}-1+\alpha} y_{n}\right\|^{1-a} \\
& =C \lambda_{n}^{a-\frac{p(1-a)}{4}}\left\|\lambda_{n}^{-1} A^{\alpha-\frac{\gamma}{2}} y_{n}\right\|^{a}\left\|\lambda_{n}^{\frac{p}{4}} A^{\frac{2-\beta+2 \alpha-\gamma}{4}-1+\alpha} y_{n}\right\|^{1-a} \\
& =C\left\|\lambda_{n}^{-1} A^{\alpha-\frac{\gamma}{2}} y_{n}\right\|^{a}\left\|\lambda_{n}^{\frac{p}{4}} A^{\frac{2-\beta+2 \alpha-\gamma}{4}-1+\alpha} y_{n}\right\|^{1-a} \\
& =o(1),
\end{aligned}
$$

where $y_{n}=\sigma A^{1-\alpha} u_{n}-m \theta_{n}$,

$$
a:=\frac{\left(\alpha-\frac{1}{2}\right)-\left(\frac{2-\beta+2 \alpha-\gamma}{4}-1+\alpha\right)}{\left(\alpha-\frac{\gamma}{2}\right)-\left(\frac{2-\beta+2 \alpha-\gamma}{4}-1+\alpha\right)}=\frac{\beta-2 \alpha+\gamma}{2+\beta-2 \alpha-\gamma},
$$


and

$$
a-\frac{p(1-a)}{4}=\frac{\beta-2 \alpha+\gamma}{2+\beta-2 \alpha-\gamma}-\frac{\beta-2 \alpha+\gamma}{2(1-\gamma)}\left(\frac{2-2 \gamma}{2+\beta-2 \alpha-\gamma}\right)=0 .
$$

The last equality in (5.19) is due to (5.14), (5.18), and $a<1$. Moreover, (5.19) implies

$$
\left\|A^{\frac{1}{2}} u_{n}\right\|=o(1)
$$

due to $\alpha-\frac{1}{2} \leq \frac{\beta}{2}$ and (5.12).

From (5.8), (5.9) we have

$$
-\rho\left\|v_{n}\right\|^{2}-\mu\left\|A^{\frac{\gamma}{2}} v_{n}\right\|^{2}+\sigma\left\|A^{\frac{1}{2}} u_{n}\right\|^{2}-m \Re\left(A^{\alpha} \theta_{n}, u_{n}\right)=o(1) .
$$

Note that, because $\alpha-\frac{\beta}{2} \leq \frac{\gamma}{2} \leq \frac{1}{2}$ in $S_{21}$,

$$
\begin{aligned}
\left|\left(A^{\alpha} \theta_{n}, u_{n}\right)\right| \leq C\left\|A^{\frac{\beta}{2}} \theta_{n}\right\|\left\|A^{\alpha-\frac{\beta}{2}} u_{n}\right\| & \leq C\left\|A^{\frac{\beta}{2}} \theta_{n}\right\|\left\|A^{\alpha-\frac{\beta}{2}} u_{n}\right\| \\
& \leq C\left\|A^{\frac{\beta}{2}} \theta_{n}\right\|\left\|A^{\frac{1}{2}} u_{n}\right\| \\
& =o(1) .
\end{aligned}
$$

Thus, we obtain from (5.21), using (5.20),

$$
\left\|v_{n}\right\|,\left\|A^{\frac{\gamma}{2}} v_{n}\right\|=o(1) .
$$

Combining (5.12), (5.20), and (5.23), we reach the promised contradiction: $\left\|U_{n}\right\|_{\mathcal{H}}=o(1)$.

Lemma 5.4. Let $p:=\frac{2(1-2 \alpha-\beta)}{1-\gamma}$. Then

$$
\limsup _{\lambda \in \mathbb{R},|\lambda| \rightarrow \infty} \frac{1}{\lambda^{p}}\left\|\left(i \lambda-\mathcal{A}_{\alpha, \beta, \gamma}\right)^{-1}\right\|_{\mathcal{L}}(H)<+\infty
$$

for $(\alpha, \beta, \gamma) \in S_{22}$.

Proof: Assume that (5.24) is false. Then, w.l.o.g. there exist a sequence $\lambda_{n} \rightarrow \infty$ and a unit sequence $U_{n}=\left(u_{n}, v_{n}, \theta_{n}\right) \in D\left(\mathcal{A}_{\alpha, \beta, \gamma}\right)$ such that $\lambda_{n}^{p}\left(i \lambda_{n} I-\mathcal{A}\right) U_{n}=F_{n}$ and

$$
\lambda_{n}^{p}\left\|\left(i \lambda_{n} I-\mathcal{A}\right) U_{n}\right\|_{\mathcal{H}}=\left\|F_{n}\right\|_{\mathcal{H}}=o(1),
$$

i.e.,

$$
\begin{array}{r}
\lambda_{n}^{p}\left(i \lambda_{n} u_{n}-v_{n}\right)=f_{1 n}=o(1), \text { in } V_{1 / 2}, \\
\lambda_{n}^{p}\left[i \lambda_{n}\left(\rho v_{n}+\mu A^{\gamma} v_{n}\right)+A^{\alpha}\left(\sigma A^{1-\alpha} u_{n}-m \theta_{n}\right)\right]=\rho f_{2 n}+\mu A^{\gamma} f_{2 n}=o(1), \text { in } V_{\gamma / 2}^{\prime}, \\
\lambda_{n}^{p}\left(\lambda_{n} c \theta_{n}+m A^{\alpha} v_{n}+k A^{\beta} \theta_{n}\right)=c f_{3 n}=o(1), \text { in } H .
\end{array}
$$

We are going to show $\left\|U_{n}\right\|_{\mathcal{H}}=o(1)$ which is a contradiction. It follows again from

$$
\Re\left(\mathcal{A}_{\alpha, \beta, \gamma} U_{n}, U_{n}\right)_{\mathcal{H}}=-k\left\|A^{\frac{\beta}{2}} \theta_{n}\right\|^{2}
$$

and (5.25) that

$$
\lambda_{n}^{\frac{p}{2}}\left\|A^{\frac{\beta}{2}} \theta_{n}\right\|=o(1) \Rightarrow\left\|\theta_{n}\right\|=o(1) .
$$


Note that $p>0$ in $S_{22}$, thus (5.14), (5.15) hold again. Since $\beta<\frac{1-\gamma}{2}$ in $S_{22}$, we have $\alpha+\beta-\frac{1}{2}<\alpha-\frac{\gamma}{2}$. Then, (5.14) implies that

$$
\left\|\lambda_{n}^{-1} A^{\alpha+\beta-\frac{1}{2}}\left(\sigma A^{1-\alpha} u_{n}-m \theta_{n}\right)\right\|=O(1) .
$$

Taking the inner product of (5.15) with $\lambda_{n}^{-1} A^{\alpha+\beta-\frac{1}{2}}\left(\sigma A^{1-\alpha} u_{n}-m \theta_{n}\right)$ in $H$, we have

$$
\begin{aligned}
o(1)= & \left(c \lambda_{n}^{\frac{p}{2}+1} A^{-\frac{\beta}{2}} \theta_{n}+m \lambda_{n}^{\frac{p}{2}+1} A^{\alpha-\frac{\beta}{2}} u_{n}, \lambda_{n}^{-1} A^{\alpha+\beta-\frac{1}{2}}\left(\sigma A^{1-\alpha} u_{n}-m \theta_{n}\right)\right) \\
= & \left(c \lambda_{n}^{\frac{p}{2}} A^{\alpha+\frac{\beta}{2}-\frac{1}{2}} \theta_{n}+m \lambda_{n}^{\frac{p}{2}} A^{2 \alpha+\frac{\beta}{2}-\frac{1}{2}} u_{n}, \sigma A^{1-\alpha} u_{n}-m \theta_{n}\right) \\
= & c \sigma\left(\lambda_{n}^{\frac{p}{2}} A^{\frac{\beta}{2}} \theta_{n}, A^{\frac{1}{2}} u_{n}\right)-c m\left\|\lambda_{n}^{\frac{p}{2}} A^{\frac{\alpha}{2}+\frac{\beta}{4}-\frac{1}{4}} \theta_{n}\right\|^{2}-m^{2}\left(A^{2 \alpha-\frac{1}{2}} u_{n}, \lambda_{n}^{\frac{p}{2}} A^{\frac{\beta}{2}} \theta_{n}\right) \\
& +m \sigma\left\|\lambda_{n}^{\frac{p}{4}} A^{\frac{1+2 \alpha+\beta}{4}} u_{n}\right\|^{2} \\
= & m \sigma\left\|\lambda_{n}^{\frac{p}{4}} A^{\frac{1+2 \alpha+\beta}{4}} u_{n}\right\|^{2}+o(1) .
\end{aligned}
$$

All terms in (5.32) are well defined since in $S_{22}$

$$
\left\{\begin{array}{l}
\alpha+\frac{\gamma}{2}-\frac{1}{2}<\frac{\beta}{2} \\
2 \alpha+\frac{\beta}{2}-\frac{1}{2} \leq 1-\alpha \\
\frac{1+2 \alpha+\beta}{4} \leq \frac{1}{2} \\
2 \alpha-\frac{1}{2} \leq \frac{1}{2} \\
\frac{\alpha}{2}+\frac{\beta}{4}-\frac{1}{4} \leq \frac{\beta}{2}
\end{array}\right.
$$

The last equality in (5.32) follows from (5.12) and the boundedness of $\left\|A^{\frac{1}{2}} u_{n}\right\|$. Therefore, (5.32) further leads to

$$
\left\|\lambda_{n}^{\frac{p}{4}} A^{\frac{1+2 \alpha+\beta}{4}-1+\alpha}\left(\sigma A^{1-\alpha} u_{n}-m \theta_{n}\right)\right\|=o(1) .
$$

because $\frac{1+2 \alpha+\beta}{4}-1+\alpha \leq 0$ in $S_{22}$.

In what follows, we consider two cases: $\beta>0$ and $\beta=0$, separately. When $\beta>0$, noting that $\frac{1}{2} \leq \frac{1}{2}+\beta<1-\frac{\gamma}{2}$ in $S_{22}$, we have

$$
\alpha-\frac{1}{2}<\alpha+\beta-\frac{1}{2}<\alpha-\frac{\gamma}{2} .
$$

Hence, by interpolation

$$
\left\|A^{\alpha+\beta-\frac{1}{2}} y_{n}\right\| \leq C\left\|A^{\alpha-\frac{\gamma}{2}} y_{n}\right\|^{\frac{2 \beta}{1-\gamma}}\left\|A^{\alpha-\frac{1}{2}} y_{n}\right\|^{1-\frac{2 \beta}{1-\gamma}},
$$

where $y_{n}=\sigma A^{1-\alpha} u_{n}-m \theta_{n}$. Since

$$
\left\|A^{\alpha-\frac{1}{2}} y_{n}\right\| \leq \sigma\left\|A^{\frac{1}{2}} u_{n}\right\|+m\left\|A^{\alpha-\frac{1}{2}} \theta_{n}\right\|=O(1)
$$

then

$$
\left\|\lambda_{n}^{-\frac{2 \beta}{1-\gamma}} A^{\alpha+\beta-\frac{1}{2}} y_{n}\right\| \leq C\left\|\lambda_{n}^{-1} A^{\alpha-\frac{\gamma}{2}} y_{n}\right\|^{\frac{2 \beta}{1-\gamma}}\left\|A^{\alpha-\frac{1}{2}} y_{n}\right\|^{1-\frac{2 \beta}{1-\gamma}}=O(1)
$$

Furthermore, note that

$$
\frac{1+2 \alpha+\beta}{4}-1+\alpha<\alpha-\frac{1}{2}<\alpha+\beta-\frac{1}{2}
$$


in $S_{22}$. Again by interpolation we get

$$
\begin{aligned}
o(1)+\left\|A^{\frac{1}{2}} u_{n}\right\| & =\left\|A^{\alpha-\frac{1}{2}} y_{n}\right\| \\
& \leq C\left\|A^{\alpha+\beta-\frac{1}{2}} y_{n}\right\|^{a}\left\|A^{\frac{1+2 \alpha+\beta}{4}-1+\alpha} y_{n}\right\|^{1-a} \\
& =C \lambda_{n}^{\frac{2 \beta a}{1-\gamma}-\frac{p(1-a)}{4}}\left\|\lambda_{n}^{-\frac{2 \beta}{1-\gamma}} A^{\alpha+\beta-\frac{1}{2}} y_{n}\right\|^{a}\left\|\lambda_{n}^{\frac{p}{4}} A^{\frac{1+2 \alpha+\beta}{4}-1+\alpha} y_{n}\right\|^{1-a} \\
& =C\left\|\lambda_{n}^{-\frac{2 \beta a}{1-\gamma}} A^{\alpha+\beta-\frac{1}{2}} y_{n}\right\|^{a}\left\|\lambda_{n}^{\frac{p}{4}} A^{\frac{1+2 \alpha+\beta}{4}-1+\alpha} y_{n}\right\|^{1-a} \\
& =o(1),
\end{aligned}
$$

where

$$
a:=\frac{\left(\alpha-\frac{1}{2}\right)-\left(\frac{1+2 \alpha+\beta}{4}-1+\alpha\right)}{\left(\alpha+\beta-\frac{1}{2}\right)-\left(\frac{1+2 \alpha+\beta}{4}-1+\alpha\right)}=\frac{1-2 \alpha-\beta}{1-2 \alpha+3 \beta}<1 .
$$

which leads to

$$
\frac{2 \beta a}{1-\gamma}-\frac{p(1-a)}{4}=\frac{2 \beta(1-2 \alpha-\beta)}{(1-\gamma)(1-2 \alpha+3 \beta)}-\frac{2 \beta(1-2 \alpha-\beta)}{(1-\gamma)(1-2 \alpha+3 \beta)}=0 .
$$

The last equality in (5.36) is due to (5.33) and (5.35).

When $\beta=0$, we still have (5.33). Since $\frac{1+2 \alpha}{4}-1+\alpha<\alpha-\frac{1}{2}<\alpha-\frac{\gamma}{2}$ in $S_{22}$, by interpolation we again obtain, using (5.14) and (5.33),

$$
\begin{aligned}
& o(1)+\left\|A^{\frac{1}{2}} u_{n}\right\|=\left\|A^{\alpha-\frac{1}{2}} y_{n}\right\| \\
& \leq C\left\|A^{\alpha-\frac{\gamma}{2}} y_{n}\right\|^{a}\left\|A^{\frac{1+2 \alpha}{4}-1+\alpha} y_{n}\right\|^{1-a} \\
& =C \lambda_{n}^{a-\frac{p(1-a)}{4}}\left\|\lambda_{n}^{-1} A^{\alpha-\frac{\gamma}{2}} y_{n}\right\|^{a}\left\|\lambda_{n}^{\frac{p}{4}} A^{\frac{1+2 \alpha}{4}-1+\alpha} y_{n}\right\|^{1-a} \\
& =C\left\|\lambda_{n}^{-1} A^{\alpha-\frac{\gamma}{2}} y_{n}\right\|^{a}\left\|\lambda_{n}^{\frac{p}{4}} A^{\frac{1+2 \alpha}{4}-1+\alpha} y_{n}\right\|^{1-a} \\
& =o(1) \text {, }
\end{aligned}
$$

where

$$
a:=\frac{\left(\alpha-\frac{1}{2}\right)-\left(\frac{1+2 \alpha}{4}-1+\alpha\right)}{\left(\alpha-\frac{\gamma}{2}\right)-\left(\frac{1+2 \alpha}{4}-1+\alpha\right)}=\frac{1-2 \alpha}{2(1-\gamma)+(1-2 \alpha)}<1,
$$

which leads to

$$
a-\frac{p(1-a)}{4}=\frac{1-2 \alpha}{2(1-\gamma)+(1-2 \alpha)}-\frac{1-2 \alpha}{2(1-\gamma)} \frac{2(1-\gamma)}{2(1-\gamma)+(1-2 \alpha)}=0 .
$$

Finally, using the same argument as in (5.21), (5.22) and the fact that $\alpha-\frac{\beta}{2}<\frac{1}{2}$, we again obtain (5.23) leading to the contradiction $\left\|U_{n}\right\|_{\mathcal{H}} \rightarrow 0$.

Lemma 5.5. For $(\alpha, \beta, \gamma) \in S_{21} \cup S_{22}$,

$$
i \mathbb{R} \subset \rho\left(\mathcal{A}_{\alpha, \beta, \gamma}\right) .
$$

Proof: Assume that (5.38) is false. Then, there exist $i \lambda \in \sigma\left(\mathcal{A}_{\alpha, \beta, \gamma}\right)$ and a unit sequence $U_{n}=\left(u_{n}, v_{n}, \theta_{n}\right) \in D\left(\mathcal{A}_{\alpha, \beta, \gamma}\right)$ such that $\left(i \lambda I-\mathcal{A}_{\alpha, \beta, \gamma}\right) U_{n}=F_{n}$ and

$$
\left\|\left(i \lambda I-\mathcal{A}_{\alpha, \beta, \gamma}\right) U_{n}\right\|_{\mathcal{H}}=\left\|F_{n}\right\|_{\mathcal{H}}=o(1),
$$


i.e.,

$$
\begin{array}{r}
\lambda_{n}^{p}\left(i \lambda_{n} u_{n}-v_{n}\right)=f_{1 n}=o(1), \text { in } V_{1 / 2}, \\
\lambda_{n}^{p}\left[i \lambda_{n}\left(\rho v_{n}+\mu A^{\gamma} v_{n}\right)+A^{\alpha}\left(\sigma A^{1-\alpha} u_{n}-m \theta_{n}\right)\right]=\rho f_{2 n}+\mu A^{\gamma} f_{2 n}=o(1), \text { in } V_{\gamma / 2}^{\prime}, \\
\lambda_{n}^{p}\left(i \lambda_{n} c \theta_{n}+k A^{\beta} \theta_{n}+m A^{\alpha} v_{n}\right)=c f_{3 n}=o(1), \text { in } H .
\end{array}
$$

Again we are going to show $\left\|U_{n}\right\|_{\mathcal{H}}=o(1)$, being a contradiction. It follows, as in the proofs of the previous lemmas,

$$
\left\|A^{\frac{\beta}{2}} \theta_{n}\right\|=o(1) \Rightarrow\left\|\theta_{n}\right\|=o(1) .
$$

Case (i): $\lambda=0$.

It follows directly from (5.40) that $\left\|A^{\frac{\gamma}{2}} v_{n}\right\|=o(1)$. Moreover, using (5.41) we have

$$
\left\|A^{\frac{1}{2}} u_{n}\right\|^{2}+m\left(A^{\alpha} \theta_{n}, u_{n}\right)=o(1) .
$$

Since $\alpha-\frac{1}{2} \leq \frac{\beta}{2}$ in $S_{21} \cup S_{22}$,

$$
\left|\left(A^{\alpha} \theta_{n}, u_{n}\right)\right| \leq\left\|A^{\frac{\beta}{2}} \theta_{n}\right\|\|\| A^{\frac{1}{2}} u_{n} \|=o(1) .
$$

Therefore, we obtain $\left\|A^{\frac{1}{2}} u_{n}\right\|=o(1)$, giving the contradiction.

Case (ii): $\lambda \neq 0$.

We can argue as in the proofs of Lemma 5.3 and Lemma 5.4, now with $p=0$.

Remark 5.6. A preliminary spectral analysis shows that there will be residual points in the spectrum on the imaginary axis for the cases when $(\alpha, \beta, \gamma)$ in $S_{2}$ with $\gamma=1$ or in $S_{3}$. It also shows that when $(\alpha, \beta, \gamma)$ is in the interior of region $S$, the real part of the eigenvalues will go to negative infinity as the imaginary part goes to infinity which is an indication for regularity of solutions. Furthermore, it also shows the optimality of the polynomial stability obtained here. These topics are under investigation.

The interesting case $\tau>0$ also asks for an investigation of polynomial stability that goes beyond the scope of the present paper and is subject to future research as well.

Acknowledgement: The paper, in particular the contributions of H.D. Fernández Sare and R. Racke, emerged from the Brazilian research project Ciências sem fronteiras.

\section{References}

[1] Ammar-Khodja, F., Bader, A., Benabdallah, A.: Dynamic stabilization of systems via decoupling techniques. ESAIM: Contr. Optim. Calc. Var. 4 (1999), 577-593.

[2] Avalos, G., Lasiecka, I.: Exponential stability of a thermoelastic system without mechanical dissipation, Rend. Instit. Mat. Univ. Trieste Suppl. 28 (1997), 1-28.

[3] Borichev, A., Tomilov, Y.: Optimal polynomial decay of functions and operator semigroups. Math. Annalen 347 (2009), 455-478. 
[4] Chen, S., Triggiani, R.: Proof of extensions of two conjectures on structural damping for elastic systems. Pacific J. Math. 136 (1989), 15-55.

[5] Danese, V., Dell'Oro, F., Pata, V.: Stability analysis of abstract systems of Timoshenko type. J. Evol. Equ. 16 (2016), 587-615.

[6] Dell'Oro, F., Muñoz Rivera, J.E., Pata, V.: Stability properties of an abstract system with applications to linear thermoelastic plates. J. Evol. Equ. 13 (2013), 777-794.

[7] R. Denk and R. Racke, $L^{p}$ resolvent estimates and time decay for generalized thermoelastic plate equations, Electronic J. Differential Equations, 48 (2006), 1-16.

[8] R. Denk, R. Racke, and Y. Shibata, $L_{p}$ theory for the linear thermoelastic plate equations in bounded and exterior domains, Advances Differential Equations, 14 (2009), 685-715.

[9] R. Denk, R. Racke, and Y. Shibata, Local energy decay estimate of solutions to the thermoelastic plate equations in two- and three-dimensional exterior domains, J. Analysis Appl., 29 (2010), 21-62.

[10] Fernández Sare, H.D., Muñoz Rivera, J.E.: Optimal rates of decay in 2-d thermoelasticity with second sound, J. Math. Phys. 53 (2012), 073509.

[11] Fernández Sare, H.D., Racke, R.: On the stability of damped Timoshenko systems - Cattaneo versus Fourier law. Arch. Rational Mech. Anal. 194 (2009), 221-251.

[12] Hao, J., Liu, Z.: Stability of an abstract system of coupled hyperbolic and parabolic equations Zeitschrift angew. Math. Phys. 64 (2013), 1145-1159.

[13] Hao, J., Liu, Z., Yong, J.: Regularity analysis for an abstract system of coupled hyperbolic and parabolic equations, J. Differential Equations 259 (2015), 4763-4798.

[14] Irmscher, T., Racke, R.: Sharp decay rates in parabolic and hyperbolic thermoelasticity, IMA J. Appl. Math. 71 (2006), 459-478.

[15] Kim, J.U.: On th energy decay of a linear thermoelastic bar and plate, SIAM J. Math. Anal. 23 (1992), 889-899.

[16] Lasiecka, I., Pokojovy, M., Wan, X.: Global existence and exponential stability for a nonlinear thermoelastic Kirchhoff-Love plate. arXiv:1612.00798v1 (2016).

[17] I. Lasiecka and R. Triggiani, Two direct proofs on the analyticity of the S.C. semigroup arising in abstract thermoelastic equations, Advances Differential Equations, 3 (1998), 387-416.

[18] I. Lasiecka and R. Triggiani, Analyticity, and lack thereof, of thermo-elastic semigroups, ESAIM, Proc., 4 (1998), 199-222.

[19] I. Lasiecka and R. Triggiani, Analyticity of thermo-elastic semigroups with coupled hinged/Neumann boundary conditions, Abstract Appl. Anal., 3 (1998), 153-169.

[20] I. Lasiecka and R. Triggiani, Analyticity of thermo-elastic semigroups with free boundary conditions, Annali Scuola Norm. Sup. Pisa, 27 (1998), 457-482.

[21] Lasiecka, I., Wilke, M.: Maximal regularity and global existence of solutions to a quasilinear thermoelastic plate system. Discr. Cont. Dyn. Sys. 33 (2013), 5189-5202.

[22] K. Liu and Z. Liu, Exponential stability and analyticity of abstract linear thermoelastic systems, Z. angew. Math. Phys., 48 (1997), 885-904. 
[23] Liu, Z., Zheng, S.: Semigroups associated with dissipative systems. Resarch Notes Math. 394, Chapman \& Hall/CRC, Boca Raton (1999).

[24] Muñoz Rivera, J.E., Racke, R.: Smoothing properties, decay, and global existence of solutions to nonlinear coupled systems of thermoelastic type, SIAM J. Math. Anal. 26 (1995), 1547-1563.

[25] Muñoz Rivera, J.E., Racke, R.: Large solutions and smoothing properties for nonlinear thermoelastic systems, J. Differential Equations 127 (1996), 454-483.

[26] Prüß, J.: On the spectrum of $C_{0}$-semigroups. Trans. Amer. Math. Soc. 284 (1984), 847-857.

[27] Quintanilla, R., Racke, R.: Addendum to: Qualitative aspects of solutions in resonators, Arch. Mech. 63 (2011), 429-435.

[28] Racke, R.: Thermoelasticity with second sound - exponential stability in linear and nonlinear 1-d, Math. Meth. Appl. Sci. 25 (2002), 409-441.

[29] R. Racke, Thermoelasticity, Chapter 4 in: Handbook of Differential Equations. Vol. 5, Evolutionary Equations. Eds.: C.M. Dafermos, M. Pokorný. Elsevier (2009), 315-420.

[30] Racke, R.: Heat conduction in elastic systems: Fourier versus Cattaneo. Proc. International Conference on Heat Transfer, Fluid Mechanics and Thermodynamics, Skukuza, South Africa (2015), $356-360$.

[31] Racke, R., Ueda, Y.: Dissipative structures for thermoelastic plate equations in $\mathbb{R}^{n}$. Differential Integral Equations 21 (2016), 601-630.

[32] Racke, R., Ueda, Y.: Nonlinear thermoelastic plate equations: global existence and decay rates for the Cauchy problem. J. Differential Equations 263 (2017), 8138-8177.

[33] Yang, X.: Generalized Form of Hurwitz-Routh criterion and Hopf bifurcation of higher order, Appl. Math. Letters 15 (2002), 615-621.

Hugo D. Fernández Sare, Department of Mathematics, Federal University of Juiz de Fora, CEP 36036900, Juiz de Fora, MG, Brazil

Zhuangyi Liu, Department of Mathematics and Statistics, University of Minnesota, Duluth, MN 558122496, USA

zliu@d.umn.edu

Reinhard Racke, Department of Mathematics and Statistics, University of Konstanz, 78457 Konstanz, Germany

reinhard.racke@uni-konstanz.de 